\section{DETERMINANTES INDIVIDUALES Y DEL ENTORNO RESIDENCIAL EN LA PERCEPCIÓN DE SEGURIDAD EN BARRIOS DEL GRAN SANTIAGO, CHILE' ${ }^{12}$}

Javier Núñez ${ }^{3}$, Ximena Tocornal ${ }^{4}$, Pablo

Henríquez ${ }^{5}$

\section{Resumen}

La percepción de seguridad de los habitantes en sus entornos residenciales más inmediatos se nutre de diversos factores tanto personales como del territorio y de las relaciones sociales entre vecinos. Este trabajo explora mediante el método estadístico de Regresión Lineal Multivariada los principales determinantes de la percepción individual de seguridad. Esta metodología se aplica con una perspectiva multinivel que considera el nivel de determinantes individuales (sexo, edad, años de educación, ingreso del hogar), como el nivel de determinantes de las relaciones sociales y del territorio residencial del individuo. Los resultados a nivel de variables individuales son coherentes con la evidencia previa. Diversas variables asociadas a la interacción social en el barrio inciden en la percepción de seguridad.

\section{INDIVIDUAL AND RESIDENTIAL SURROUNDING DETERMINANTS ON THE SENSE OF SECURITY IN NEIGHBORHOODS OF SANTIAGO, CHILE $^{12}$}

Javier Núñez ${ }^{3}$, Ximena Tocornal ${ }^{4}$, Pablo Henríquez $^{5}$

\begin{abstract}
:
The sense of security of inhabitants within the immediate residential surrounding is nurtured by personal and land factors and social relationships among neighbors. By using multivariate linear regression, this paper explores the main factors that determine the individual perception of security. This methodology is applied with a multilevel perspective that considers individual determinants (age, education, household income) and determinants regarding social relationships and the residential area of the individual. The results at variable level are consistent with previous evidence. Different variables related to social interaction within a neighborhood have impact on the sense of
\end{abstract}


En particular, confianza e interacción entre vecinos junto a la antigüedad de los barrios son relevantes en la determinación de la percepción de seguridad. El rol de las señales de desorden físico no parece relevante. La presencia de programas de seguridad ciudadana a nivel municipal en el territorio es significativa, indicando la relevancia de estas iniciativas en frenar el temor de las comunidades.

PALABRAS CLAVE: SEGURIDAD, TEMOR AL DELITO, ENTORNO RESIDENCIAL, RELACIONES VECINALES.

Fecha de recepción: 15.11.11

Fecha de aceptación: 23.03.12

1 Este trabajo es parte del Proyecto ANILLOS de Investigación en Ciencias Sociales denominado "Crimen y Violencia Urbana. Aportes de la teoría ecológica del delito al diseño de políticas públicas", financiado por el Comisión Nacional de Investigación Científica y Tecnológica (CONICYT) del Gobierno de Chile, desarrollado por un grupo interdisciplinario de investigadores de la Universidad de Chile y la Universidad de Santiago de Chile.

2 Los autores agradecen las sugerencias de los colegas del equipo Hugo Fruhling, Mauricio Olavarría, Ricardo Tapia y Roberto Gallardo, además de aquellas recibidas durante la Conferencia Internacional Violencia en Barrios en América Latina, realizada el 5 y 6 de octubre de 2011 en la Facultad de Economía y Negocios de la Universidad de Chile, organizada por el Centro de Estudios en Seguridad Ciudadana (CESC) del Instituto de Asuntos Públicos y el Instituto de la Vivienda (INVI) de la Facultad de Arquitectura y Urbanismo, ambos de la Universidad de Chile.

3 Chile. Departamento de Economía, Facultad de Economía y Negocios, Universidad de Chile.

4 Chile. Centro de Estudios en Seguridad Ciudadana, Instituto de Asuntos Públicos, Universidad de Chile.

5 Chile. Facultad de Economía y Negocios, Universidad de Chile. security. Particularly, trust and interaction among neighbors and the age of neighborhoods are important elements that determine the sense of security. The role played by physical disorder does not seem relevant. The presence of security programs at municipal level is important as it reveals the relevance of these initiatives.

\section{KEYWORDS: SECURITY, FEAR OF CRIME, RESIDENTIAL SURROUNDING, NEIGHBORLY RELATIONSHIPS.}

Received: 15.11 .11

Accepted: 23.03.12

1 This paper is part of the ANILLOS Social Sciences Research Project "Crime and Urban Violence. Contributions of Ecology Theory to the Design of Public Policies" funded by CONICYT and developed by an interdisciplinary team of researchers from Universidad de Chile and Universidad de Santiago de Chile.

2 The authors of this paper thank the suggestions made by Hugo Frühling, Mauricio Olavarría, Ricardo Tapia and Roberto Gallardo and those received at the International Conference on Violence on Latin American Neighborhoods, held at the Facultad de Economía y Negocios, Universidad de Chile on October 5-6, 2011 and organized by the Centro de Estudios de Seguridad Ciudadana, Instituto de Asuntos Públicos and the Instituto de la Vivienda, Facultad de Arquitctura y Urbanismo, Universidad de Chile.

3 Chile. Departamento de Economía, Facultad de Economía y Negocios, Universidad de Chile.

4 Chile. Centro de Estudios en Seguridad Ciudadana, Instituto de Asuntos Públicos, Universidad de Chile.

5 Chile. Facultad de Economía y Negocios, Universidad de Chile. 


\section{Introducción}

La percepción de (in)seguridad constituye una de las principales preocupaciones de la ciudadanía, así como también de las autoridades de gobierno local y nacional. Sin embargo, la investigación y el conocimiento respecto de los determinantes de la percepción de seguridad es aún limitado y fragmentario y, en particular, muy poco se sabe aún respecto del rol que juegan las características ambientales y sociales de los entornos residenciales para influir en las percepciones de seguridad de las personas. En este contexto, este trabajo busca aportar al conocimiento sobre esta materia, al estudiar el rol diferenciado de dimensiones individuales (tales como edad, género, experiencias pasadas de victimización y nivel de ingresos por hogar) y de dimensiones sociales y territoriales del entorno residencial de los individuos.

Un desafío particular que enfrenta esta investigación emana del hecho de que las percepciones de seguridad pueden estar influidas de manera directa por las características de la interacción social en el barrio, pero también por la prevalencia de experiencias de victimización recientes ocurridas dentro del entorno residencial, lo cual puede también estar asociado a las características de la interacción social dentro de éste. Esto plantea, en principio, un potencial doble efecto de los atributos de la interacción social dentro del barrio sobre la percepción individual de seguridad que abordamos por medio del desarrollo de un método multinivel.

El trabajo emplea una base de datos de 242 entornos residenciales (o barrios) del Gran Santiago, basado en respuestas de 5860 individuos. El cuestionario incluye diversas preguntas. La base de datos fue complementada con una pauta de observación para recoger atributos observables y objetivos del entorno físico y social de los barrios. A pesar de la riqueza de las dimensiones individuales y de los entornos incluidos en la base de datos, se debe considerar que ésta corresponde a un corte transversal, de manera que los resultados y conclusiones del presente estudio deben tomarse con la cautela que exigen estudios de esta naturaleza.

El artículo está estructurado como sigue: la segunda sección presenta una revisión de la literatura relacionada, que proporciona el contexto para las hipótesis abordadas por este trabajo. La tercera sección presenta la metodología utilizada en el estudio para evaluar las hipótesis del trabajo. La sección cuarta presenta los datos que se utilizan. La quinta sección presenta y discute los resultados obtenidos. Finalmente, la sección sexta concluye. 


\section{Revisión de literatura relacionada}

En la literatura especializada acerca de la percepción de seguridad o inseguridad, con respecto a la amenaza que representa la posibilidad de ser víctima de un delito para las personas en contextos urbanos, no existe consenso en cuanto a la definición conceptual del fenómeno, así como tampoco con respecto a su medición operacional ${ }^{6}$. De hecho, la mayoría de los autores que hacen una revisión exhaustiva del tema ${ }^{7}$, así como aquellos que lo estudian empíricamente analizando los efectos de variables no individuales en el nivel de temor al delito, insisten en esta falta de parámetros conceptuales y metodológicos comunes y la necesidad de contar con más estudios empíricos. Las variables no personales estudiadas empíricamente son tan diversas como, por ejemplo, el tipo de viviendas que componen el barrio ${ }^{8}$, características de la política social de los países $^{9}$, la eficacia colectiva y la cultura

$6 \quad$ Esta falta de consensos se nutre también del hecho de que se hable indistintamente del fenómeno en términos de nivel de percepción de seguridad o inseguridad o de miedo o temor al delito, cuando cada uno de estos términos connota tradiciones distintas en las ciencias sociales y en la medición de fenómenos psicológicos y psicosociales.

7 Por ejemplo, Vozmediano, San Juan y Vergara, 2008; Dammert, 2007; Pain, 2000

8 Villata, 2011.

9 Hummelsheim, Hirtenlehner, Jackson y Oberwittler, 2011. ciudadana ${ }^{10}$, las relaciones informales entre vecinos y el nivel del desorden físico y social percibido en el entorno residencial ${ }^{11}$. No obstante, sí hay acuerdo en sostener que ciertas variables personales sociodemográficas, como el género y la edad, son factores que influyen en el nivel de temor al delito ya que se ha encontrado evidencia consistente de que las mujeres experimentan mayores niveles de temor al delito que los hombres y, con evidencia un poco menos generalizada, que a mayor edad, mayores niveles de temor al delito. Por su parte la variable de nivel de ingreso económico de los hogares, ha mostrado ser significativa en algunos estudios ${ }^{12}$ de tal modo que a mayores ingresos, se evidencian niveles de temor al delito más alto. No obstante, también existen estudios cualitativos ${ }^{13}$ que señalan que no sólo serían los sectores socioeconómicos más elevados aquellos con más temor al delito, sino que este fenómeno tiene gran relevancia y características particulares entre los sectores menos aventajados debido a su mayor vulnerabilidad y percepción de exclusión social. Por lo tanto, el nivel de ingresos económicos (asociado a nivel educacional u otro

10 Ruiz, 2010

11 Brunton-Smith y Sturgis, 2011; Roccato, Russo y Vieno, 2011; Ross y Jang, 2000.

12 Pain, 2000; Hummelsheim, Hirtenlehner, Jackson y Oberwittler 2011.

13 Dammert, 2004 
similar), el género y la edad suelen ser variables de control insoslayables en los estudios cuantitativos.

Los estudios empíricos que miden el nivel de inseguridad o temor al delito aparecen en la década de los 80, específicamente en el Reino Unido a partir de la sistematicidad con que se empiezan a aplicar encuestas de victimización. Al respecto, es importante destacar que las encuestas de victimización son construidas con la finalidad de entregar información útil a la toma de decisiones de política pública $^{14}$, más que con fines investigativos académicos que descansen en definiciones decantadas de un determinado fenómeno social ${ }^{15}$.

Con respecto a la variabilidad de ítems o reactivos que se utilizan en las encuestas, los más comúnmente utilizados $-\mathrm{y}$ sus variantes menores- son: 1) "¿qué tan seguro se siente usted caminando solo en su barrio cuando es de noche?", dando por alternativas de respuesta una gradiente de Escala Likert de entre 3 a 5 posibilidades (de muy seguro a muy inseguro); 2) "iusted considera que en los últimos 12 meses, la delincuencia en su barrio/ciudad/país ha aumentado, se ha mantenido o a disminuido?” y 3) “cuán

14 Pain, 2000.

15 Ello podría explicar en parte, la variabilidad de reactivos utilizados para medir el fenómeno, en tanto operazionalizaciones de un constructo inacabado debido a la urgencia por medirlo, predecirlo e intervenirlo, siendo prácticamente la redacción del ítem lo que determine la definición conceptual. Otra posibilidad es afirmar que el fenómeno es especialmente complejo y sus definiciones, diversas, ambiguas e inacabadas. probable es que usted sea víctima de un delito en los próximos 12 meses?", también con alternativas que van entre 3 a 5 posibilidades (de muy probable a muy improbable). Si bien estos reactivos son tratados como si midieran el mismo fenómeno, es posible constatar sutilezas entre ellos, como que la primera alternativa no hace mención a la delincuencia $y$, por ende, podría estar recogiendo una evaluación de riesgos un poco más amplia que las dos siguientes, que sí acotan la situación consultada a la delincuencia. Pero aun así, es probable que no sea tan simple aislar tanto en su definición conceptual como en su operacionalización para la investigación, la medición del temor a la delincuencia de otros tipos de temores más difusos y característicos de las sociedades postmodernas contemporáneas asociados a situaciones tales como la pérdida de referentes y certezas valóricas e ideológicas, el mundo globalizado y las interconexiones e interdependencias entre lo local y lo global ${ }^{16}$. Diversos problemas sociales y económicos "tales como la vivienda, el trabajo, la planificación del espacio y la exclusión social (relacionada con pobreza, género, raza y similares)"17, estarían

16 Hummelsheim, Hirtenlehner, Jackson y Oberwittler, 2011.

17 Pain, 2000, p. 365 
configurando que en la consulta por el temor al delito también sean capturados otros temores asociados a la vida en las grandes urbes contemporáneas. No es casual, entonces, que en las políticas públicas haya un giro hacia estrategias de reducción del temor desde lo más local reduccionista hacia aproximaciones más holísticas ${ }^{18}$, lo que pone en evidencia, finalmente, el carácter complejo del fenómeno del temor al delito y la inseguridad, más allá de las discusiones sobre su medición.

A partir de lo anterior, es posible argumentar que mediciones del temor al delito y la inseguridad ciudadana como un todo pueden ser mejor capturadas con reactivos más amplios y que no nombren explícitamente la delincuencia, como el ya mencionado "¿qué tan seguro se siente usted caminando solo en su barrio cuando es de noche?" y otras variantes ${ }^{19}$.

Ahora bien, hay consenso entre los estudiosos del tema con respecto a la diferencia sustancial entre temor a distintos tipos de delitos. La personas temen de manera diferenciada y experimentan distintas emociones y evaluaciones de riesgo ante la eventualidad de ser víctimas de un delito con

18 Pain, 2000.

19 Autores como Vozmediano, San Juan y Vergara (2008) prefieren una definición más precisa del fenómeno, entendido como miedo al delito. Ellos argumentan que las medidas de intensidad (aquellas que van de mucho a poco) así como los ítems de qué tanto, qué tan probable favorecen la sobreestimación. Por ende sugieren acotar el marco temporal y espacial usando medidas de frecuencia. violencia hacia su integridad física o la de sus seres queridos, que ante la posibilidad de ser víctimas de la extracción o daño de un bien. Por ello, se recomienda ampliamente consultar por niveles de temor de manera específica para diversos tipos de delitos, según se trate de delitos contra la propiedad o contra las personas, sin o con violencia. Sin embargo, también es cierto que muchas veces un mismo hecho delictual implica todas las categorías anteriores o, al menos, pueden estar implicados en las representaciones o imágenes de la delincuencia que se activan en los sujetos cuando se les consulta $^{20}$. Finalmente, estas representaciones se nutren -y retroalimentan cual círculo vicioso- de los discursos de los medios de comunicación y de los discursos políticos acerca de la delincuencia y la seguridad.

Si bien hay ciertos matices entre las definiciones de percepción de in/seguridad ciudadana y miedo o temor al delito ${ }^{21}$, todas ellas intentan dar cuenta de las reacciones emocionales afectivas -y sus correlatos conductuales- ante la posibilidad de ser objeto de un ataque a la integridad física en la vía pública o en el hogar, que la propiedad privada sea

20 Pain, 2000.

21 La literatura inglesa, utiliza "fear of crime", traducible como miedo o temor al delito. Dammert (2007) privilegia "temor" mientras que Vozmediano, San Juan y Vergara (2008) y Ruiz (2010) utilizan "miedo". En psicología general miedo es una noción más específica que temor (Bruno, 1997). Por ende, utilizar miedo privilegia un enfoque más específico sobre la amenaza que representa la delincuencia. 
violentada y/o extraída, o de que otras personas cercanas sufran experiencias de esa índole. Para Ross y Jang ${ }^{22}$, el temor al delito no es solo una respuesta individual; es la consecuencia de la erosión del control social. Para Vozmediano, San Juan y Vergara $^{23}$, el miedo al delito es "una experiencia de naturaleza emocional, suscitada por la posibilidad de ser víctima de un delito" y en tanto tal "es el resultado de una determinada manera de procesar la información a partir de elementos que nos proporciona el entorno" tales como noticias, rumores, discursos políticos, lo que dará lugar a respuestas conductuales como la disminución de uso de espacios públicos, posiciones punitivas con respecto a cómo enfrentar el delito, discriminación o xenofobia. En suma, el empobrecimiento de la vida social o la reproducción del círculo vicioso al que se hizo mención. La gran mayoría de estudios empíricos y reflexiones acerca de la relación entre seguridad y calidad de vida están de acuerdo en este punto, en cómo el temor al delito genera consecuencias negativas en varias dimensiones de la vida cotidiana, la economía y la política ${ }^{24}$.

Vilalta ${ }^{25}$, en un intento por sistematizar los estudios que abordan este fenómeno proponiendo variables asociadas al menos a nivel de correlaciones,

22 Ross y Jang, 2000.

23 Vozmediano, San Juan y Vergara, 2008, p. 7 .

24 Crawford, 1999.

25 Vilalta, 2011.

ARTÍCULO: Determinantes individuales y del entorno residencial en la percepción de seguridad en barrios del Gran Santiago, Chile / Javier Núñez, Ximena Tocornal, Pablo Henríquez los agrupa en dos niveles de análisis: la comunidad y el individuo; lo que a su vez le permite organizar las distintas teorías del temor al delito en 5 grupos: 1) la probada influencia de las experiencias de victimización personal y vicarias ${ }^{26}$ en el temor al delito; 2) la evaluación de vulnerabilidad física en función de características como edad y género; 3) la vulnerabilidad social de ciertos grupos que evidencian mayor exposición a riesgos y amenazas variadas (grupos marginados); 4) el desorden social en tanto facilitador de delito y del temor al delito; y 5) el doble rol que juegan las redes sociales en tanto pueden explicar el aumento y el decremento de los niveles de temor al delito cuando ello se pone en relación con otras dimensiones de la vida social. Mientras 1 y 2 son teorías del nivel individual, 3, 4 y 5 corresponden al nivel de la comunidad. Esta investigación se detendrá particularmente en ellas ya que también son mencionadas en otras investigaciones relevantes para efectos de este trabajo.

Esta estrategia de distinguir entre factores individuales y otros de carácter social, los que a su vez pueden ser divididos entre aquellos del contexto residencial más inmediato o del barrio y otros más amplios aún, es una forma de agrupar los factores

26 La victimización vicaria es aquella que se produce cuando un individuo conoce de cerca una situación de victimización delictual a través de los relatos de amigos o familiares cercanos, produciéndose la identificación y la apropiación de la experiencia sin haberla vivido experimentado las emociones asociadas a ésta.

revista invi № 74 / Mayo 2012 / Volumen № 27: 87-120 
asociados al temor al delito bastante común en la literatura y en los modelos de análisis empleados. Ello parece razonable en la medida de que se trata de un fenómeno que se experimenta individuamente (la ansiedad, la emoción) pero que tiene una raíz y un correlato social indiscutible.

A continuación se revisan someramente algunos estudios empíricos recientes sobre los efectos de factores de la comunidad en el nivel de temor al delito o inseguridad de las personas. Esta categoría de "lo comunitario" agrupa dimensiones en rigor no individuales, a pesar de que para observarlas haya que consultar a los individuos particulares, como han sido definidas en la tradición de investigación que se desprende de los estudios de Robert Sampson y sus colegas ${ }^{27}$. Estos autores se han dedicado desde comienzos de los '90 a investigar los factores de las comunidades asociados a la violencia y la delincuencia, más que el temor al delito. No obstante, los conceptos que ellos han refinado, como lazos sociales, eficacia colectiva, control social informal y otros, han sido testeados por otros investigadores en relación con el temor al delito.

27 Sampson, Raudenbush y EARLS, 1997; Raudenbush y Sampson 1999; Sampson y Raudenbush, 1999; Sampson, Mcadam, Macindoe, Weffer-Elizondo y Civil, 2005.
En general, el nivel de la comunidad designa una serie de atributos del conjunto (más que de la suma de las partes), como cualidades de las dinámicas relacionales entre vecinos y percepciones compartidas que definen la identidad del sector residencial, a partir de sus características físicas concretas como de sus correlatos simbólicos. Así, por ejemplo, la literatura señala que los espacios poco cuidados con señales de desorden físico, falta de iluminación adecuada ${ }^{28}$, así como espacios públicos que no permiten la visibilidad afectan los niveles de temor al delito de las personas, pero más importante que ello es la influencia de los lazos o vínculos sociales ("social ties", en inglés) informales entre los vecinos ${ }^{29}$.

Rosso y Jang ${ }^{30}$ sostienen que los lazos sociales ejercen un efecto amortiguador del grado de desorden percibido en el vecindario, sobre los niveles de temor al delito y de desconfianza. El punto de partida de estos autores es que existe suficiente evidencia de que a mayor percepción de señales de desorden físico (autos abandonados, jardines mal cuidados, calles en mal estado, mobiliario público destruido, por ejemplo) y de desorden social (personas

28 Con respecto a la iluminación en la vía pública, la evidencia es contradictoria. En algunos casos, se asocia a menos temor (más visibilidad, más control del espacio, menos temor) y en otros a más temor (más luz, más posibilidades de ser visto por eventuales delincuentes y atacado, más temor). Pain, 2000.

29 Pain, 2000; Ross y Jang, 2000.

30 Ross y Jang, 2000. 
deambulando en el sector sin una finalidad, uso de lenguaje grotesco, discusiones, por ejemplo), se experimenta mayor percepción de temor al delito, recociendo también que las experiencias de victimización son muy relevantes, incluso más que la percepción de desorden.

En base a una encuesta telefónica realizada en 1995 a 2484 encuestados mayores de 18 años de la ciudad de Illinois, Estados Unidos, los autores concluyen que los efectos del desorden percibido en el barrio en el temor y la desconfianza no son tan importantes cuando la integración informal entre vecinos es alta. El nivel de integración informal es consultado a través de preguntas tales como qué tan seguido usted visita a sus vecinos, conversa con ellos y se ayudan entre sí (en situaciones como préstamos de herramientas, por ejemplo).

Las conclusiones del estudio de Ross y Jang señalan que los efectos del desorden percibido sobre el temor y la desconfianza son condicionados por la integración informal con los vecinos. Mientras más alto es el desorden percibido, más alto es el nivel de importancia de las variables individuales y menos las de las vínculos sociales. O sea, la integración no puede neutralizar los efectos del desorden percibido sobre el temor al delito, solo los amortigua o disminuye. En barrios donde el desorden percibido es alto, los individuos que refieren mayor nivel de integración formal, tienen niveles de temor más bajos que aquellos que se quedan aislados. Pero en lugares donde el desorden percibido es promedio o bajo el promedio, los lazos sociales no tienen efectos significativos sobre el temor al delito. Además, este estudio confirma que las mujeres y los no blancos tienen más temor al delito. La presencia de niños en el hogar resulta importante. La edad no aparece significativa. Ajustando por desorden percibido e integración informal, el nivel educacional y de ingresos no están significativamente asociados al temor al delito. De modo tal que, más importante que las variables individuales (salvo género y raza), las variables de contexto del barrio, el desorden percibido y los lazos sociales en este caso, son las más relevantes para explicar el temor al delito de las personas encuestadas.

Por su parte, un estudio hecho por Roccato, Russo y Vieno ${ }^{31}$ en base a una encuesta aplicada en Italia en 2008, indica que el nivel de desorden media los efectos de las experiencias de victimización en el temor al delito. Se afirma que la victimización indirecta o vicaria (aquella de algún conocido o familiar) refuerza el temor al delito entre aquellas personas que reportaron percibir mayor desorden social en sus comunidades. Sin embargo, si se habita en un sector donde no se perciben altos niveles de desorden social, entonces ni la victimización directa o indirecta tienen efectos sobre el temor al delito.

31 Roccato, Russo y Vieno, 2011. 
Otra investigación relevante es la de BruntonSmith y Sturgis ${ }^{32}$ que utiliza varias aplicaciones de la British Crime Survey y otros datos como variables demográficas de los barrios, signos visuales de desorden físico y niveles de delincuencia reportadas por los vecinos. En síntesis, estos investigadores reportan que estos tres grupos de variables, características estructurales de los vecindarios, niveles de desorden físico y victimización tienen efectos sobre el nivel de temor al delito.

Finalmente, cabe destacar los resultados de la investigación de Ruiz ${ }^{33}$ en Bogotá, Colombia. Se trata de un estudio con una muestra intencionada de 400 sujetos a los que se les aplicó un complejo instrumento que incluye variadas mediciones para eficacia colectiva, confianza, cultura ciudadana, participación local, afecto por la ciudad, respeto a las normas legales, clima emocional, satisfacción con la policía y victimización, además de complejas formas de medir el miedo al delito en distintos contextos y ante situaciones delictivas específicas. Las variables que se utilizan como controles son edad, género, estrato socioeconómico, nivel educativo -desde primaria a posgrado-, y composición del hogar.

Los resultados de esta investigación indican que "la cultura ciudadana y la eficacia colectiva

32 Brunton-Smith y Sturgis, 2011.

33 Ruiz, 2010

96 revista invi № 74 / Mayo 2012 / Volumen N ${ }^{0}$ 27: 87-120 aparecen muy relacionadas, pero es esta última, específicamente la dimensión de unión de la comunidad, la que guarda mayor relación con los niveles de miedo al crimen" 34 . Es decir, si bien hay una alta correlación entre la cultura ciudadana y la eficacia colectiva, es la dimensión de unión o cohesión social entre los vecinos la que efectivamente se asoció con menores niveles de miedo al delito. En general, el temor es menos importante cuando se trata de ámbitos socio-geográficos sobre los cuales el sujeto tiene un contacto más directo y prolongado -hogar y barrio-. Por último, se señala que, a nivel de correlaciones, hay una asociación entre nivel socioeconómico más bajo y mayor temor al delito, lo que se explica debido a que las personas de ingresos más bajos también habitan los sectores de la ciudades en condiciones comparativamente más deficientes en cuanto a iluminación nocturna, estado de las calles, acceso a servicios de agua, gas o electricidad, grado de estabilidad y condiciones de trabajo, entre otras, que podrían explicar los índices más elevados en el miedo al delito.

En síntesis, a pesar de los esfuerzos por definir conceptualmente el temor al delito y la inseguridad en las ciudades de hoy, aún subsiste la pregunta sobre la especificidad de este fenómeno y sus formas de medición. Si como señalan Hummelsheim,

34 Ruiz, 2010, p. 112 
Hirtenlehner, Jackson, y Oberwittler ${ }^{35}$ el temor al delito es "una respuesta no solamente frente a la percepción del problema del delito, sino también a la forma, textura y salud atribuible a las estructuras políticas y sociales", entonces cobra pleno sentido estudiar los determinantes sociales del temor al delito en los sujetos individuales, en la medida de que éstos son parte de estructuras sociales y políticas que se experimentan en la cotidianidad en los entornos residenciales más inmediatos. Es así como los recursos de la comunidad como un conjunto, la capacidad del cuerpo social para controlar las señales de desorden físico y social, así como las cualidades de las interacciones informales y recurrentes, pueden configurarse y estudiarse como aquellos recursos simbólicos y concretos, a los que el individuo puede recurrir para convivir de manera más saludable, menos ansiógena y restrictiva frente a las amenazas de las formas de vida actuales, algunas supuestamente más objetivas o indiscutibles que otras pero, al fin y al cabo, todas ellas efectivamente experimentadas por los sujetos.

\section{Metodología del estudio}

Este trabajo se basa en el método estadístico de Regresión Lineal Multivariada para analizar los principales determinantes de la percepción individual de seguridad en los entornos residenciales. Esta metodología se aplica con una perspectiva multinivel, es decir, considera tanto el nivel de ciertos determinantes individuales, como también el nivel de determinantes propios del entorno residencial del individuo.

Sobre la base de la discusión previa de la literatura relacionada, es posible distinguir tres grupos principales de variables que en principio influenciarían la percepción de seguridad de un individuo por distintas vías. Es así como el modelo general está dado por la siguiente ecuación:

$$
y=\alpha+\underset{a}{\beta X_{a}+\gamma X_{b}+\delta X_{c}+\varepsilon}
$$

En la ecuación (1), podemos distinguir las siguientes variables:
35 Hummelsheim, Hirtenlehner, Jackson, Y Oberwittler, 2011, p. 328. 
Y es la variable dependiente, es decir, la percepción de seguridad ${ }^{36}$ del individuo en su barrio.

$\boldsymbol{\alpha}$ es la constante del modelo de regresión lineal.

$\boldsymbol{X}_{a}$ es el grupo de variables sociodemográficas personales del individuo (sexo, edad, años de educación y nivel de ingresos del hogar) más las experiencias recientes de victimización personal (número de delitos de los que fue víctima la persona, diferenciado si éstos fueron en el sector residencial o fuera de él, en los últimos 12 meses). Llamaremos a esta agrupación de variables Grupo A.

$\boldsymbol{X}_{\boldsymbol{b}}$ agrupa un conjunto de variables sociales y territoriales del barrio. En primer lugar, siguiendo la literatura desarrollada por Sampson y otros investigadores, se integran aquellas variables relacionadas con la convivencia entre vecinos y los recursos asociados a ella (confianza, cohesión, contactos informales y control social informal). En segundo lugar, se agrupan variables tales como la evaluación que hacen los vecinos de la presencia de instituciones del Estado en el territorio (municipios, salud y educación). Y finalmente, se agregan las variables señales de desorden físico y presencia de comercio de primera necesidad en el barrio. Llamaremos a esta agrupación

36 Hay discusión sobre la terminología adecuada para denominar esta variable. En particular, dada la pregunta de la encuesta que se está ocupando en este trabajo, parece más apropiado referirse a percepción de seguridad ya que el ítem no menciona directamente "delincuencia". No obstante, en lo que sigue se utilizarán temor al delito y percepción de seguridad de manera indistinta. de variables Grupo C. Este ordenamiento obedece al efecto que pueda tener este grupo de variables sobre el Grupo $C$ y no necesariamente a una tipología establecida en función de una naturaleza común a todas las variables incluidas en el Grupo B.

$\boldsymbol{X}_{c}$ incluye las variables del barrio tasa de victimización por delitos ocurridos adentro del territorio, percepción de violencia e incivilidades promedio de los habitantes del barrio. Se espera que estas variables estén directamente relacionadas con la percepción de seguridad, ya que mientras más situaciones violentas e incivilidades ocurran y sean percibidas por el conjunto de residentes, es lógico pensar que las personas se sientan más inseguras dentro de éste. Llamaremos a esta agrupación de variables Grupo C.

$\boldsymbol{\varepsilon}$ es el error aleatorio del modelo, el cual recoge todos aquellos elementos aleatorios que no pueden ser controlados ni observados.

La figura 1 señala cómo los tres grupos de variables interactúan hipotéticamente entre sí para afectar la percepción de seguridad individual. Como se indica, es esperable que las variables del Grupo $\mathrm{C}$ asociadas a niveles de delito y percepción de violencia

7757 Revista Invi 74 Interior indb 98 


\section{FIGURA 1: MODELO CONCEPTUAL GENERAL}

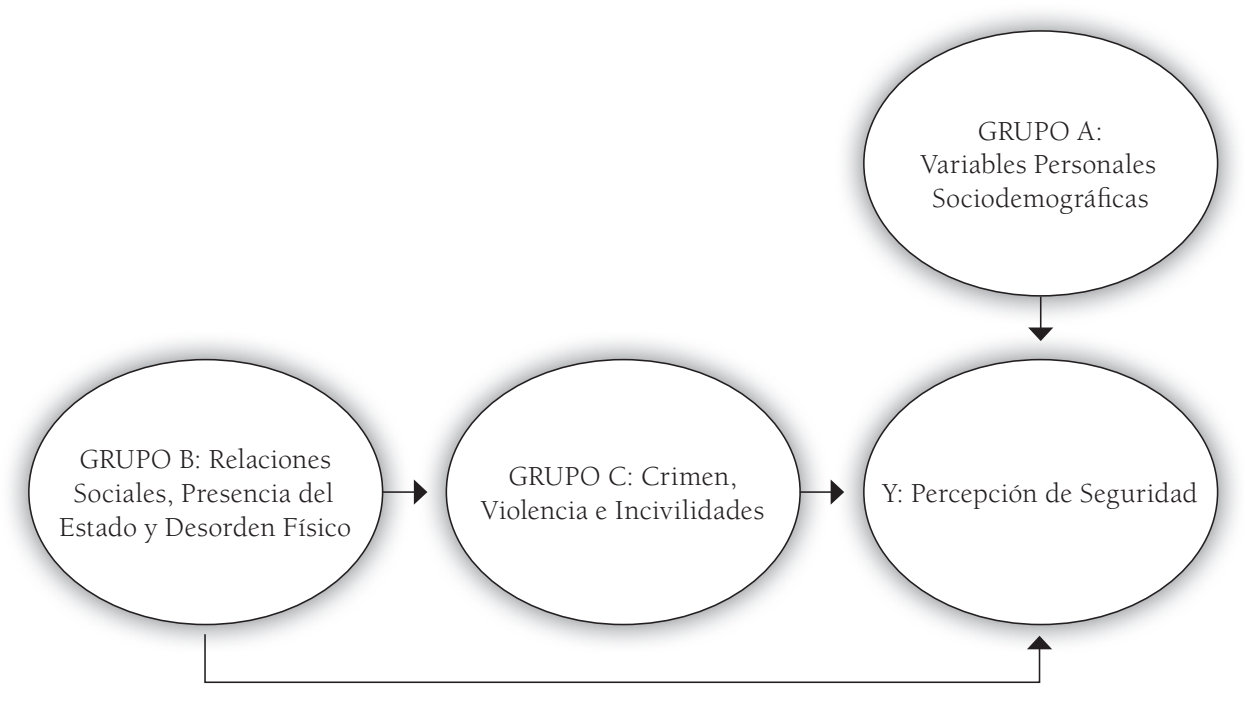

e incivilidades tengan un efecto directo sobre la percepción de seguridad individual en el entorno residencial, toda vez que este grupo de variables recoge la percepción compartida y socializada de cuán relevante serían para el conjunto de los residentes las amenazas delictuales y violentas experimentadas en el barrio. Por su parte, el Grupo B de variables puede tener simultáneamente un efecto directo y uno indirecto sobre la percepción de seguridad individual. El efecto directo podría estar dado por cómo las variables de este grupo, esto es, redes de colaboración informal entre vecinos, la mayor cohesión social y confianza entre ellos, así como la presencia de algunas instituciones del Estado (programas de seguridad preventiva a nivel municipal) y de la vida social (comercio de primera necesidad, por ejemplo), pueden afectar directamente la sensación de seguridad individual en la medida en que se evalúa contar con mayores recursos de ayuda mutua en diversas eventualidades, incluida la amenaza de la violencia y la delincuencia. Por otra parte, el efecto indirecto de las variables del Grupo B sobre la percepción de seguridad individual estaría dado por cómo estas variables tendrían efectos sobre la 
victimización y la percepción de violencia e incivilidades en el barrio (las variables del Grupo C), lo que a su vez afectaría la percepción de seguridad. En otras palabras, el efecto indirecto dice relación con cómo comunidades más cohesionadas y con mayores recursos institucionales establecerían las acciones coordinadas formal e informalmente para disminuir los eventos delictuales y/o violentos, reduciendo las variables del Grupo C.

Este efecto doble de las variables del Grupo B puede generar problemas de endogeneidad en las estimaciones debido a que dentro del mismo modelo, algunas variables que explican la variable dependiente (la percepción de seguridad individual), también tendrían un efecto causal sobre algunas variables explicativas, pudiendo sesgar así los coeficientes. Por ejemplo, es posible suponer que la variable cohesión social en el barrio tiene efectos en la percepción de seguridad y al mismo tiempo afecta la ocurrencia de incivilidades, las que a su vez también influyen en la percepción de inseguridad. La literatura econométrica ha discutido en profundidad métodos para resolver este tipo de problemas. No obstante, en este caso particular se enfrentan algunas dificultades que invalidan muchos de estos mecanismos. En primer lugar, la base de datos a utilizar es, hasta ahora, una encuesta de corte transversal, por lo cual todos los esquemas de análisis en diferencias quedan descartados. En segundo lugar, no fue posible encontrar instrumentos con buenas propiedades estadísticas, por lo cual el esquema de variables instrumentales no fue utilizado.

\section{FIGURA 2: MODELO A}

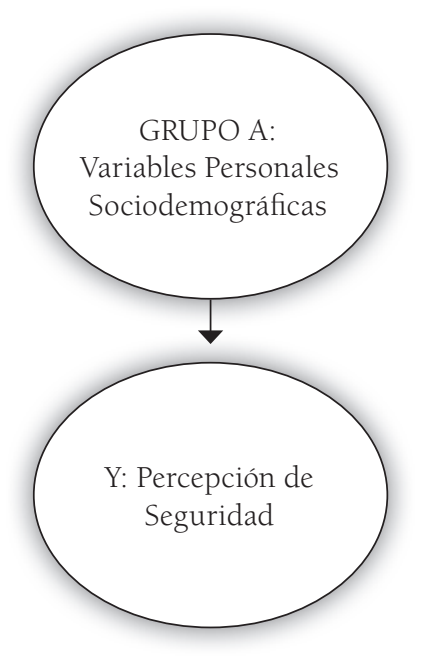

Entendiendo que este es un modelo general, se usarán también versiones restringidas del mismo, los cuales incluyen uno o dos grupos de variables. Esta forma de operar se utiliza para aislar los posibles efectos causales y mecanismos de transmisión entre grupos de variables explicativas.

Es así como comenzamos por el Modelo A (ver figura 2), el modelo más sencillo, que sólo incluye las variables personales sociodemográficas del Grupo A y analiza cómo éstas afectan la percepción de seguridad, por sí solas. Así logramos despejar el rol de las características inherentes a la persona. Estas variables se utilizarán como controles en todas las especificaciones siguientes. El modelo queda entonces definido por la siguiente ecuación: 


\section{FIGURA 3: MODELO AC}

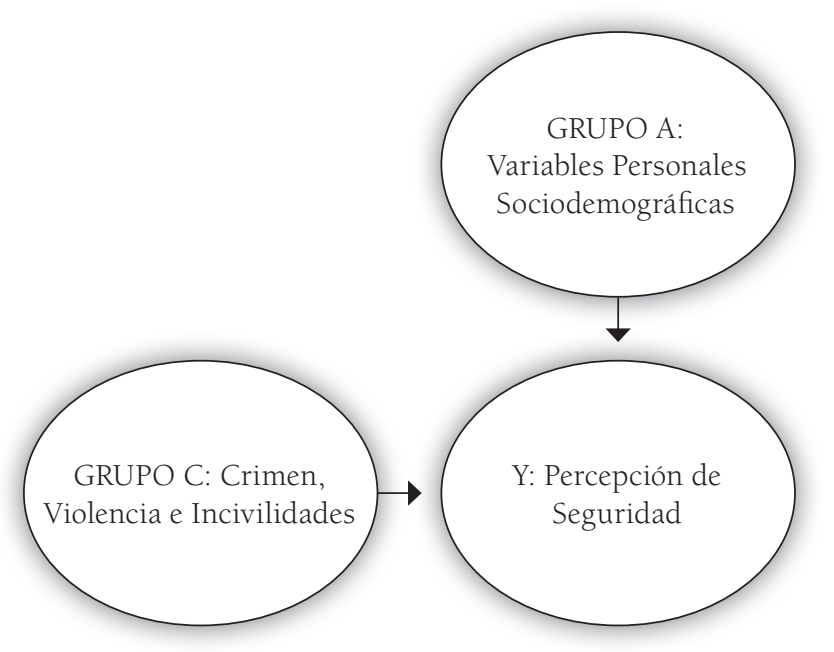

$$
y=\alpha+\beta X_{a}+\varepsilon
$$

Luego, agregamos a las variables del Grupo A, aquellas del Grupo C que dicen relación con violencia, crimen e incivilidades en el barrio, conformando el Modelo Ac (ver figura 3). Ello permite analizar el efecto que tienen las variables del Grupo $C$ sobre la variable a explicar en ausencia de las variables del Grupo B, controlando por las variables sociodemográficas individuales del Grupo A. Este modelo responde a la ecuación:

$$
y=\alpha+\beta X_{a}+\delta X_{c}+\varepsilon
$$

ARTíCULO: Determinantes individuales y del entorno residencial en la percepción de seguridad en barrios del Gran Santiago, Chile / Javier Núñez, Ximena Tocornal, Pablo Henríquez

\section{FIGURA 4: MODELO AB}

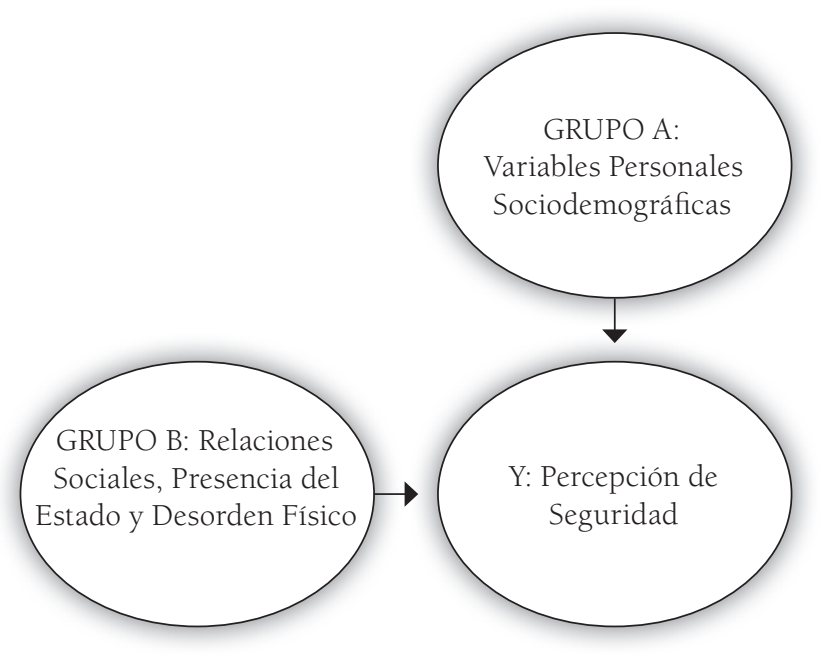

El siguiente modelo AB (ver figura 4) es aquel en el cual se combinan las variables sociodemográficas individuales del Grupo A y las del nivel comunitario del Grupo B. Este tercer modelo permite aislar el efecto de las variables del Grupo B con respecto a las variables del Grupo C. Este modelo responde a laecuación:

$$
y=\alpha+\beta X_{a}+\gamma X_{b}+\varepsilon
$$

Y finalmente el Modelo ABC responde a la figura 1 , en el cual se agregan los 3 grupos de variables, respondiendo a la ecuación general:

$$
y=\alpha+\beta X_{a}+\gamma X_{b}+\delta X_{c}+\varepsilon
$$

revista invi № 74 / Mayo 2012 / Volumen № 27: 87-120 101 


\section{Datos}

Este trabajo utiliza una encuesta a 5860 hogares del Santiago de Chile, en el contexto del Proyecto ANILLOS de Investigación en Ciencias Sociales, denominado "Crimen y Violencia Urbana. Aportes de la teoría ecológica del delito al diseño de políticas públicas". La encuesta fue aplicada entre agosto y octubre de 2010 en 242 barrios del Gran Santiago. En cada barrio, se encuestaron cerca de 24 a 25 hogares en promedio, seleccionados de manera aleatoria para obtener representatividad al interior del barrio.

La muestra del estudio fue obtenida de la siguiente manera. Primero, se seleccionaron al azar 121 Unidades Vecinales ${ }^{37}$ (UV) sobre un universo de 885 UV en las 34 comunas urbanas de Santiago. Luego, por cada UV seleccionada se eligieron al azar 2 conjuntos residenciales de 6 manzanas censales ${ }^{38}$

37 La Unidad Vecinal es una división político-administrativa del territorio de la comuna, cuyo gobierno local está en manos del Alcalde Municipal. Las Unidades Vecinales tienen por finalidad estimular la organización de los vecinos y permitir la expresión de intereses ante el municipio y otras autoridades del Estado. La Junta de Vecinos es la denominación que se da a la organización ciudadana de ese espacio subcomunal, regulada por la ley 19.418. En el Gran Santiago existen 885 UV.

38 La manzana es la unidad territorial más básica con que opera el CENSO poblacional en Chile según el Instituto Nacional de Estadísticas (INE). Corresponde al espacio territorial interior comprendido en la intersección de tres o más calles, dibujando normalmente un cuadrado o rectángulo, sin que haya en este espacio una unidad menor que responda a esta misma definición. en promedio, unidad que fue creada especialmente para efectos de este estudio, que recibió la denominación de MicroBarrios (MB). Para establecer el universo de MBs, se construyó una grilla de MBs sobre las 34 comunas urbanas de la ciudad, mediante un procedimiento de iteración matemática que respetó hitos urbanos relevantes (por ejemplo, ningún $\mathrm{MB}$ puede incluir una avenida o carretera importante que eventualmente signifique la división del barrio en dos conjuntos residenciales separados de facto). Así se conformó un universo de 8.206 MBs. En un tercer momento, por cada uno de los MBs seleccionados, se encuestaron cerca de 25 hogares $^{39}$, mediante un procedimiento de saltos aleatorios entre viviendas por calle. Cuando un hogar seleccionado aleatoriamente no pudo ser entrevistado, se reemplazó por otro seleccionado de manera aleatoria dentro del mismo MB, hasta alcanzar una meta referencial de 25 hogares por MB (o eventualmente hasta agotar los hogares del

39 El número de 25 hogares fue impuesto como una meta referencial para cada microbarrio, debido a que es un número que permite obtener estadísticos (promedios a nivel de MB, por ejemplo) con buenas propiedades estadísticas (varianzas pequeñas, acotadas), bajo el supuesto de que dichos hogares son seleccionados de manera aleatoria. 
$\mathrm{MB})$, meta que se cumplió en la gran mayoría de los casos, siendo el promedio resultante de hogares por MB de 24,2. En promedio, los MBs tienen cerca de 200 hogares y comprenden una población de 824 habitantes distribuidos en una superficie de 57.291 metros cuadrados.

En la figura 5 se muestra la distribución espacial en el Gran Santiago de los MB seleccionados mediante el procedimiento señalado. No obstante, es importante destacar que la muestra no tiene representatividad comunal o por zonas de la ciudad, sino que para Santiago en su conjunto, es decir, los 242 MBs y las 121 UVs encuestados representan a todos los MBs y las UVs de las comunas urbanas de Santiago. ${ }^{40}$

La encuesta está estructurada en 5 módulos, más uno que consulta las características sociodemográficas del encuestado y del jefe de hogar. El primer módulo incluye información básica sobre el hogar. El segundo, recoge la percepción del encuestado acerca de características relevantes del entorno residencial (elección del barrio, estabilidad residencial, satisfacción con el barrio, actividades rutinarias, trasporte público, entre otras). El tercer

40 Dado que Santiago es una ciudad espacialmente segregada en materia socioeconómica, la dispersión geográfica de los microbarrios logra representar de manera adecuada a los diversos niveles socioeconómicos del Gran Santiago. El análisis empírico que sigue en la Sección V, hace uso de diversas medidas socioeconómicas de los microbarrios como factores asociados o explicativos de las hipótesis analizadas en este estudio. módulo consulta sobre presencia y efectividad del Estado (presencia y uso de instituciones de educación y salud públicas y privadas, relación con los municipios, confianza en instituciones de control formal, entre otras). El cuarto módulo recoge las percepciones del encuestado en cuanto a los recursos comunitarios que identifica en su barrio (confianza, cohesión, frecuencia de contactos, calidad de los vínculos en el contexto barrial, participación vecinal, control social informal, identidad y adhesión a valores y normas prosociales comunes, entre otras). El último módulo de victimización delictual, violencia e incivilidades, consulta sobre eventos delictuales sufridos por el hogar y el encuestado -siguiendo las formas tradicionales de las encuestas de victimización- más preguntas sobre percepción de violencia e incivilidades en el barrio.

Además en el marco del proyecto mencionado, un grupo de investigadores especialmente entrenados realizaron observaciones sistemáticas en los 242 barrios donde se aplicó la encuesta. Mediante una pauta estandarizada se registraron un conjunto de indicadores relevantes para la seguridad en los entornos residenciales, tales como presencia de 


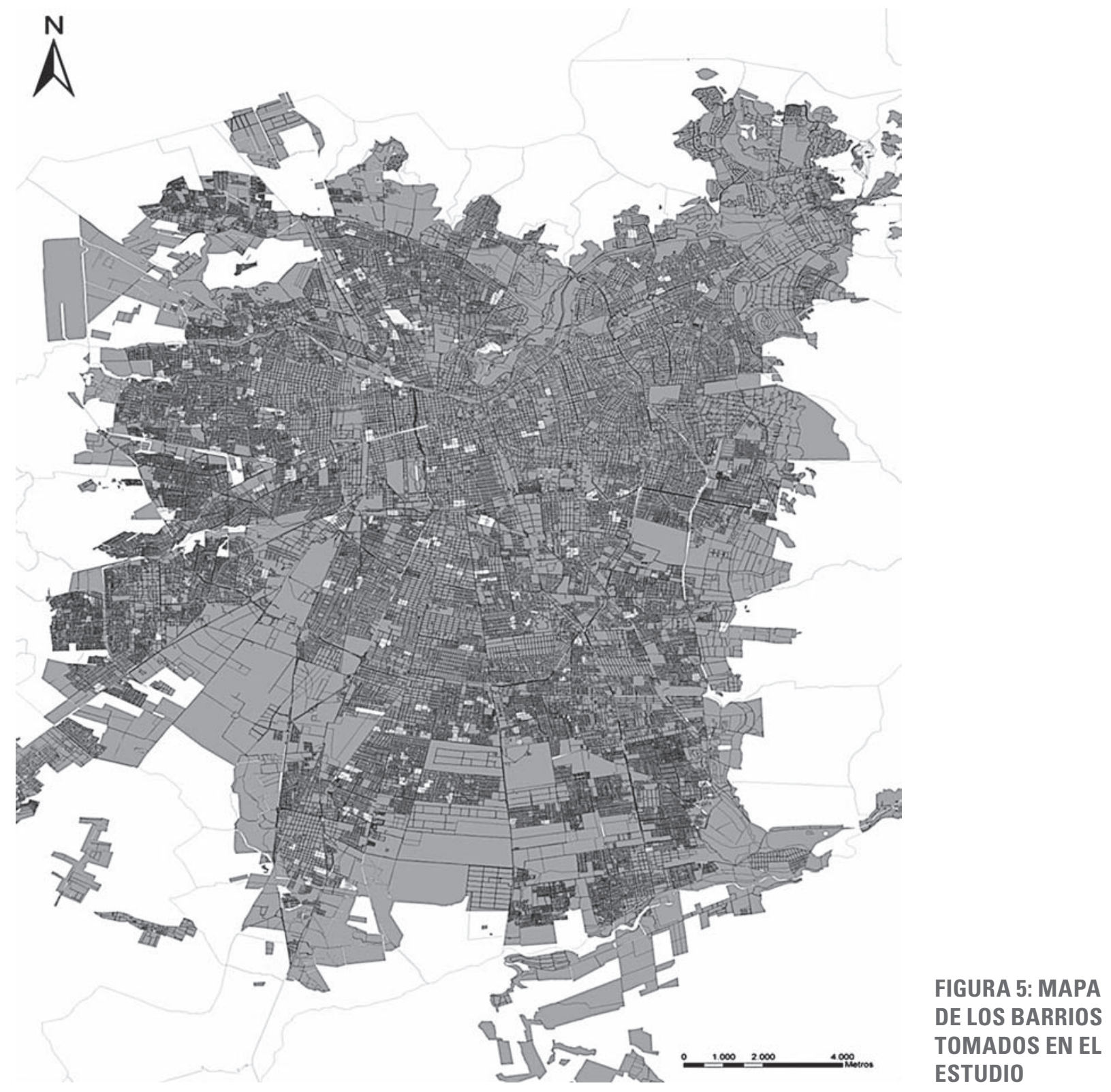

104 revista invi № 74 / Mayo 2012 / Volumen № 27: 87-120

ARTíCULO: Determinantes individuales y del entorno residencial en la percepción de seguridad en barrios del Gran Santiago, Chile / Javier Núñez, Ximena Tocornal, Pablo Henríquez 
espacios inseguros (sitios eriazos, callejones, etc.), señales de desorden físico (presencia de basura, botellas de alcohol vacías en calles y parques, autos abandonados, etc.), señales de desorden social (ruidos molestos, personas deambulando, uso de lenguaje grosero, discusiones en la vía pública, etc.), presencia de instituciones públicas y privadas (jardines infantiles, colegios, centros médicos, etc.) y presencia de comercio de primera necesidad y servicios, entre otros.

A continuación se detallan las características de las variables que específicamente se utilizan en este trabajo. La variable dependiente Y percepción de seguridad del encuestado con respecto a su barrio es una agregación de 4 preguntas en Escala Likert de la encuesta: Qué tan seguro se siente (donde 1 es muy seguro y 5 muy inseguro) ante las siguientes situaciones: 1) caminando solo por el barrio de día, 2) esperando el transporte público en el barrio, 3) cuando un desconocido ronda por el barrio, 4) caminando solo por el barrio cuando anochece.

Las variables del Grupo A sexo, edad, años de educación y número de experiencias de victimización delictual en el barrio y fuera de éste, son del entrevistado, mientras que el ingreso corresponde al hogar del entrevistado.

Las variables del Grupo B y del Grupo C corresponden todas al MB del entrevistado. Algunas fueron construidas, según sea el caso, en base a promedios individuales que agrupan varios ítems que miden una misma variable que luego fueron promediados a nivel del MB. Enseguida, se asignó este promedio del MB a cada uno de los integrantes del respectivo barrio.

En particular, la medida de percepción de violencia para el MB se obtuvo en base a las respuestas individuales bajo formato de Escala Likert, consultando con qué frecuencia de 1 a 5 (donde 1 es nunca y 5 es siempre) ocurren las siguientes situaciones de violencia: 1) Amenazas y agresiones contra transeúntes; 2) Asaltos a transeúntes con violencia o intimidación; 3) Amenazas y riñas entre grupos; 4) Apropiación de espacios públicos por personas dedicadas a cometer delitos y/o al tráfico de drogas; 5) Destrucción de la propiedad privada; 6) Destrucción de la infraestructura pública; 7) Robos en viviendas o vehículos.

Para la medida de percepción de incivilidades del MB se utiliza el mismo procedimiento, consultando por las siguientes situaciones: 1) Lenguaje agresivo en la vía pública; 2) Daños a la infraestructura pública; 3) Apropiación de plazas o sitios eriazos por grupos que provocan desórdenes; 4) Consumo de alcohol o drogas en la vía pública; 5) Acumulación de basura y falta de higiene en el entorno; 6) Ruidos molestos.

Para la tasa de victimización del MB se considera el porcentaje de hogares por MB donde alguno de sus miembros ha sido víctima de un delito dentro del barrio en los últimos 12 meses. 
Con respecto a las variables del Grupo B, salvo las variables de antigüedad del $M B$ (que corresponde al promedio de años de residencia en la misma vivienda), desorden físico MB y presencia de comercio de primera necesidad (que se obtienen de la pauta de observación), el resto siguen el mismo procedimiento de la variable de percepción de violencia del MB.

Para las medidas de confianza del MB se tomó la pregunta: ¿En cuántos de sus vecinos usted podría confiar?, en escala de 1 a 5 , donde 1 es ninguno y 5 es muchos.

Para las medidas de cohesión del MB se tomaron las respuestas ante la pregunta: ¿Cuán de acuerdo está con las afirmaciones? (de 1 a 5 donde 1 es totalmente en desacuerdo y 5 es totalmente de acuerdo): 1) Los vecinos de este barrio son muy unidos; 2) nos comunicamos y entendemos entre los vecinos; 3) se actúa con solidaridad y colaboración entre los vecinos; 4) los vecinos del barrio no comparten los mismos valores; 5) se producen conflictos entre los vecinos. Cabe destacar que las últimas dos afirmaciones fueron invertidas para que quedaran como atributos positivos que contribuyeran a mayor cohesión barrial.

Para las medidas de interacción informal del $M B$ se utilizaron las respuestas a: ¿Con qué frecuencia diría usted que se dan las siguientes situaciones en el barrio? (donde 1 es nunca y 5 es siempre): 1) Los vecinos se saludan; 2) los vecinos conversan unos con otros; 3) los vecinos hacen amistades entre sí;
4) los vecinos intercambian favores cuando lo necesitan (problemas de salud, empleo, otros); 5) los vecinos suelen visitar a otros en sus casas.

Para las medidas de disposición a actuar en comunidad del MB se utilizaron las respuestas a: ¿Con qué frecuencia diría usted que los vecinos se organizan para los siguientes fines o situaciones? (donde 1 es nunca y 5 es siempre): 1) Para transportarse en un auto común; 2) celebrar navidad, fiestas patrias u otras; 3) cuidado o limpieza de plazas y calles; 4) cuidar o vigilar el entorno; 5) ayudar a un vecino cuando tiene un problema.

Para las medidas de control social informal del MB se consultó por la probabilidad de que un vecino intervenga cuando (donde 1 es muy improbable y 5 muy probable): 1) jóvenes hacen la cimarra; 2) un vecino anda por las calles ebrio; 3) hay una pelea frente a su casa; 4) un vecino grita o golpea a su hijo en público; 5) vecinos botan basura o dañan mobiliario urbano; 6) hay venta o consumo de drogas en los espacios públicos; 7) un desconocido intenta robar a algún vecino.

En este Grupo B también se incluyen otras variables del territorio relevantes para la seguridad personal. Estas son la calificación promedio del MB que obtienen los programas municipales de seguridad ciudadana y una evaluación de cuán probable creen los individuos del barrio que lleguen ambulancias de SAMU, carabineros, policía de investigaciones y bomberos, variable denominada Acuden 
TABLA 1: EFECTOS DE VARIABLES PERSONALES SOCIODEMOGRÁFICAS (GRUPO A) EN PERCEPCIÓN DE SEGURIDAD

\begin{tabular}{|c|c|c|c|}
\hline & \multicolumn{3}{|c|}{ Modelo } \\
\hline Variables & A1 & $\mathrm{A} 2$ & A3 \\
\hline Victimización individual en el barrio & $\begin{array}{r}-0.2852^{* * *} \\
(0.0000)\end{array}$ & $\begin{array}{r}-0.2863^{* * *} \\
(0.0000)\end{array}$ & $\begin{array}{r}-0.2819^{* * *} \\
(0.0000)\end{array}$ \\
\hline Victimización individual fuera del barrio & $\begin{array}{r}-0.4565^{* * *} \\
(0.0008)\end{array}$ & $\begin{array}{r}-0.4713^{* * *} \\
(0.0005)\end{array}$ & $\begin{array}{r}-0.4398^{* * *} \\
(0.0013)\end{array}$ \\
\hline Sexo & $\begin{array}{r}1.01991^{* * *} \\
(0.0000) \\
\end{array}$ & $\begin{array}{r}1.05618^{* * *} \\
(0.0000) \\
\end{array}$ & $\begin{array}{r}1.01142^{* * *} \\
(0.0000) \\
\end{array}$ \\
\hline Educación & $\begin{array}{r}0.01310 \\
(0.4240)\end{array}$ & $\begin{array}{l}- \\
-\end{array}$ & $\begin{array}{r}0.02682^{*} \\
(0.0770)\end{array}$ \\
\hline Edad & $\begin{array}{r}-0.0071^{* *} \\
(0.0277)\end{array}$ & $\begin{array}{r}-0.0081^{* * *} \\
(0.0055)\end{array}$ & - \\
\hline Ingreso & $\begin{array}{r}0.0000010^{* * *} \\
(0.0000)\end{array}$ & $\begin{array}{r}0.0000011^{* * *} \\
(0.0000)\end{array}$ & $\begin{array}{r}0.0000010^{* * *} \\
(0.0000)\end{array}$ \\
\hline Constante & $\begin{array}{r}11.9006 \\
(0.0000) \\
\end{array}$ & $\begin{array}{r}12.0373 \\
(0.0000) \\
\end{array}$ & $\begin{array}{r}11.4177 \\
(0.0000) \\
\end{array}$ \\
\hline $\begin{array}{l}\text { Número Observaciones } \\
R_{a}^{2}\end{array}$ & $\begin{array}{r}3939 \\
0.0433 \\
\end{array}$ & $\begin{array}{r}4111 \\
0.0459 \\
\end{array}$ & $\begin{array}{r}3939 \\
0.0423 \\
\end{array}$ \\
\hline
\end{tabular}

NOTA: Los números entre paréntesis corresponden a los p-value de los coeficientes estimados en la regresión. Los otros valores corresponden a los coeficientes de la regresión lineal, los cuales muestran en cuánto se ve afectada la variable dependiente (percepción de seguridad) ante variaciones en la(s) variables explicativas. Los espacios en blanco corresponden a variables que no fueron incluidas en el modelo respectivo.

*** Estadísticamente significativo al 1\%

"* Estadísticamente significativo al 5\%

"Estadísticamente significativo al 10\% 
MB. Asimismo, se incluyen variables sobre la satisfacción promedio del MB con los servicios públicos de salud y educación presentes en el barrio.

\section{Resultados}

\section{MODELO A: REGRESIONES CON SOLO LAS CARACTERÍSTICAS SOCIODEMOGRÁFICAS PERSONALES.}

La tabla 1 muestra los resultados de la estimación por Mínimos Cuadrados Ordinarios (MCO) del modelo restringido que sólo incluye variables personales sociodemográficas. Al analizarla podemos verificar que la gran mayoría de las variables personales tienen un rol significativo en los niveles de percepción de seguridad individual.

Es posible constatar que la percepción de seguridad en el barrio disminuye al aumentar el número de delitos del que ha sido víctima el entrevistado, sin importar si éstos ocurrieron dentro o fuera del entorno residencial más inmediato. Asimismo, el género tiene un rol importante, siendo las mujeres quienes tienden a reportar un menor nivel de seguridad en su barrio. Los años de educación resultan no ser significativos, mientras que la edad sí lo es, por lo que a mayor edad, tenemos personas más inseguras. Por su parte, a mayor ingreso económico del hogar, los entrevistados se sienten más seguros.

\section{MODELO AC: REGRESIONES CON \\ CARACTERÍSTICAS SOCIODEMOGRÁFICAS \\ PERSONALES Y DELITOS, VIOLENCIA E INCIVILIDADES EN EL BARRIO.}

La tabla 2 muestra los resultados de las estimaciones por MCO del modelo restringido que agrega a las variables individuales aquellas que dicen relación con la percepción de violencia e incivilidades en el barrio más la tasa de victimización por delitos ocurridos dentro de éste. Nótese que las variables del Grupo C son calculadas como promedios para cada barrio y luego imputadas a cada entrevistado según el barrio donde habite ya que lo que interesa en este trabajo es explicar una variable individual como es la percepción de seguridad de las personas en sus entornos residenciales inmediatos.

El número de delitos del que ha sido víctima el individuo tanto dentro como fuera del barrio continúan siendo variables significativas que hacen disminuir la percepción de seguridad. No obstante, el valor del coeficiente para el caso de la victimización fuera del barrio baja con respecto al modelo anterior, es decir, la victimización fuera del barrio resulta menos importante cuando en el modelo se incluyen las variables de percepción del MB de violencia, incivilidades más la tasa de victimización de delitos ocurridos en este territorio.

Las otras variables control como sexo, edad y nivel de ingresos del hogar en este modelo AC se comportan de la misma manera que en el modelo simple 
TABLA 2: EFECTOS DE VARIABLES PERSONALES SOCIODEMOGRÁFICAS (GRUPO A) Y DE DELITOS, VIOLENCIA E INCIVILIDADES (GRUPO C) EN PERCEPCIÓN DE SEGURIDAD.

\begin{tabular}{|c|c|c|c|c|}
\hline \multirow{2}{*}{ Variables } & & \multicolumn{3}{|c|}{ Modelo } \\
\hline & & $\mathrm{AC} 1$ & $\mathrm{AC2}$ & AC3 \\
\hline \multirow[t]{3}{*}{ Variables Grupo C } & Incivilidades MB & $\begin{array}{r}0.08282^{* * * *} \\
(0.0022)\end{array}$ & $\begin{array}{r}-0.1372^{* * *} \\
(0.0000)\end{array}$ & \\
\hline & Violencia MB & $\begin{array}{r}-0.1494^{* * *} \\
(0.0000)\end{array}$ & - & $\begin{array}{r}-0.1048^{* * *} \\
(0.0000)\end{array}$ \\
\hline & Victimización MB & $\begin{array}{r}-4.5719^{* * *} \\
(0.0000)\end{array}$ & $\begin{array}{r}-4.0372^{* * *} \\
(0.0000)\end{array}$ & $\begin{array}{r}-4.1720^{* * *} \\
(0.0000)\end{array}$ \\
\hline \multirow[t]{9}{*}{ Variables Grupo A } & Victimización individual en el barrio & $\begin{array}{r}-0.2313^{* * *} \\
(0.0000)\end{array}$ & $\begin{array}{r}-0.2290^{* * *} \\
(0.0000)\end{array}$ & $\begin{array}{r}-0.2336^{* * * *} \\
(0.0000)\end{array}$ \\
\hline & Victimización individual fuera del barrio & $\begin{array}{r}-0.4023^{* * *} \\
(0.0023)\end{array}$ & $\begin{array}{r}-0.3846^{* * *} \\
(0.0036)\end{array}$ & $\begin{array}{r}-0.3955^{* * *} \\
(0.0024)\end{array}$ \\
\hline & Sexo & $\begin{array}{r}0.99428^{* * *} \\
(0.0000) \\
\end{array}$ & $\begin{array}{r}1.01744^{* * *} \\
(0.0000) \\
\end{array}$ & $\begin{array}{r}1.00474^{* * *} \\
(0.0000) \\
\end{array}$ \\
\hline & Educación & $\begin{array}{r}-0.0148 \\
(0.3591) \\
\end{array}$ & $\begin{array}{r} \\
- \\
\end{array}$ & - \\
\hline & Edad & $\begin{array}{r}-0.0145^{* * *} \\
(0.0000)\end{array}$ & $\begin{array}{r}-0.0124^{* * *} \\
(0.0000)\end{array}$ & $\begin{array}{r}-0.0133^{* * *} \\
(0.0000)\end{array}$ \\
\hline & Ingreso & $\begin{array}{r}0.0000004^{* *} \\
(0.0419)\end{array}$ & $\begin{array}{r}0.0000004^{* *} \\
(0.0298)\end{array}$ & $\begin{array}{r}0.0000003^{*} \\
(0.0752)\end{array}$ \\
\hline & Constante & $\begin{array}{r}15.5129^{* * *} \\
(0.0000)\end{array}$ & $\begin{array}{r}15.3523^{* * *} \\
(0.0000)\end{array}$ & $\begin{array}{r}15.6325^{* * *} \\
(0.0000)\end{array}$ \\
\hline & Número Observaciones & 3939 & 4111 & 4111 \\
\hline & $R_{a}^{2}$ & 0.1070 & 0.0865 & 0.1055 \\
\hline
\end{tabular}

NOTA 1: Las variables terminadas en MB son promedios a nivel de microbarrio, las que no incluyen dicha clasificación son variables individuales del entrevistado.

NOTA 2: Los números entre paréntesis corresponden a los p-value de los coeficientes estimados en la regresión, los otros valores corresponden a los coeficientes de la regresión lineal, los cuales muestran en cuánto se ve afectada la variable dependiente (percepción de seguridad) ante variaciones de la variable explicativa. Los espacios en blanco corresponden a variables que no fueron incluidas en el modelo respectivo.

$* * * *$ Estadísticamente significativo al $1 \%$

"Estadísticamente significativo al 5\%

"Estadísticamente significativo al 10\% 
restringido, esto es, son variables significativas para explicar la percepción de seguridad en el barrio, donde mujeres, personas de mayor edad y entrevistados de menores ingresos económicos tienen menor percepción de seguridad. No obstante llama la atención que el nivel de significancia del ingreso del hogar baja de un 1\% en el modelo anterior a un 5\% o 10\% dependiendo de la combinación de regresores.

En cuanto a las nuevas variables, es importante destacar que la percepción de incivilidades y de violencia en el barrio son colineales, ya que al agregar ambas variables como regresores del modelo, los coeficientes tienen signos contra-intuitivos y diferentes a los que se obtienen al agregarlas por separado (ver regresión AC2 y AC3). Además entre percepción de incivilidades y de violencia en el barrio existe una correlación de 0.79 (medida de correlación de Pearson). En este sentido, cada una de las variables por separado son significativas en términos de que a mayor incivilidad o violencia, menor percepción de seguridad.

Finalmente, la tasa de victimización por delitos ocurridos en el barrio es significativa, de tal forma que a mayor victimización en el barrio, menor percepción de seguridad.

\section{MODELO AB: REGRESIONES CON \\ CARACTERÍSTICAS SOCIODEMOGRÁFICAS PERSONALES Y VARIABLES SOCIALES Y TERRITORIALES DEL BARRIO}

La Tabla 3 muestra los resultados de la estimación MCO del tercer tipo de modelo restringido, el cual incorpora una serie de variables calculadas como promedio a nivel del barrio. Como ya se mencionó, las variables del Grupo B, al igual que las del Grupo C, son valores promedios de las barrios, imputados a los individuos de acuerdo al barrio donde habiten. Además, las variables del Grupo B son de diversa índole cubriendo aquellas del nivel comunitario según los estudios de Sampson, las de presencia del Estado, señales de desorden físico y presencia de comercio de primera necesidad.

La victimización personal, tanto dentro como fuera del barrio, continúan afectando de manera negativa y significativa la percepción de seguridad en la medida en que se introducen nuevas variables al modelo. Cabe destacar que la magnitud de los coeficientes de ambas variables se ha mantenido estable a través de los diversos modelos probados. 
TABLA 3: EFECTOS DE VARIABLES PERSONALES SOCIODEMOGRÁFICAS (GRUPO A) Y DE VARIABLES SOCIALES Y TERRITORIALES (GRUPO B) EN PERCEPCIÓN DE SEGURIDAD.

\begin{tabular}{|c|c|c|c|c|c|c|}
\hline \multirow{2}{*}{\multicolumn{2}{|c|}{ Variables }} & \multicolumn{5}{|c|}{ Modelo } \\
\hline & & AB1 & AB2 & AB3 & AB4 & AB5 \\
\hline \multirow[t]{12}{*}{$\begin{array}{l}\text { Variables } \\
\text { Grupo B }\end{array}$} & Antigüedad MB & $\begin{array}{r}0.02792^{* * *} \\
(0.0009)\end{array}$ & $\begin{array}{r}0.02824^{* * * *} \\
(0.0005)\end{array}$ & $\begin{array}{r}0.02897^{* * *} \\
(0.0004)\end{array}$ & $\begin{array}{r}0.02878^{* * *} \\
(0.0004)\end{array}$ & $\begin{array}{r}0.03379^{* * *} \\
(0.0000)\end{array}$ \\
\hline & Confianza MB & $\begin{array}{c}0.70192^{* * * *} \\
(0.0000)\end{array}$ & $\begin{array}{r}0.64725^{* * *} \\
(0.0000)\end{array}$ & $\begin{array}{r}0.59581^{* * *} \\
(0.0000)\end{array}$ & $\begin{array}{r}0.56717^{* * *} \\
(0.0000)\end{array}$ & $\begin{array}{r}0.60700^{* * * *} \\
(0.0000)\end{array}$ \\
\hline & Cohesión MB & $\begin{array}{r}0.10325^{* *} \\
(0.0384)\end{array}$ & $\begin{array}{r}0.09809^{* *} \\
(0.0432)\end{array}$ & $\begin{array}{r}0.11379^{* *} \\
(0.0154)\end{array}$ & $\begin{array}{r}0.10954^{* *} \\
(0.0196)\end{array}$ & - \\
\hline & $\begin{array}{l}\text { Disp. Actuar en Comunidad } \\
\text { MB }\end{array}$ & $\begin{array}{r}-0.0459^{* *} \\
(0.0349)\end{array}$ & $\begin{array}{r}-0.0451^{* *} \\
(0.0328)\end{array}$ & $\begin{array}{r}-0.0445^{* *} \\
(0.0345)\end{array}$ & - & - \\
\hline & Interacción MB & $\begin{array}{r}0.06723^{* *} \\
(0.0172)\end{array}$ & $\begin{array}{r}0.07222^{* * * *} \\
(0.0080)\end{array}$ & $\begin{array}{r}0.07986^{* * *} \\
(0.0032)\end{array}$ & $\begin{array}{r}0.05301^{* *} \\
(0.0265)\end{array}$ & $\begin{array}{r}0.06956^{* * *} \\
(0.0023)\end{array}$ \\
\hline & Control Social Informal MB & $\begin{array}{r}-0.0669^{* * *} \\
(0.0000)\end{array}$ & $\begin{array}{r}-0.0688^{* * *} \\
(0.0000)\end{array}$ & $\begin{array}{r}-0.0661^{* * *} \\
(0.0000)\end{array}$ & $\begin{array}{r}-0.0725^{* * *} \\
(0.0000)\end{array}$ & $\begin{array}{r}-0.0664^{* * *} \\
(0.0000)\end{array}$ \\
\hline & Seguridad Ciudadana MB & $\begin{array}{r}0.58807^{* * *} \\
(0.0000) \\
\end{array}$ & $\begin{array}{r}0.60257^{* * *} \\
(0.0000) \\
\end{array}$ & $\begin{array}{r}0.68032^{* * *} \\
(0.0000) \\
\end{array}$ & $\begin{array}{r}0.64801^{* * *} \\
(0.0000) \\
\end{array}$ & $\begin{array}{r}0.69898^{* * *} \\
(0.0000) \\
\end{array}$ \\
\hline & Acuden MB & $\begin{array}{r}0.22459^{* * *} \\
(0.0000) \\
\end{array}$ & $\begin{array}{r}0.21807^{* * *} \\
(0.0000) \\
\end{array}$ & $\begin{array}{r}0.22609^{* * *} \\
(0.0000) \\
\end{array}$ & $\begin{array}{r}0.23287^{* * *} \\
(0.0000) \\
\end{array}$ & $\begin{array}{r}0.23730^{* * *} \\
(0.0000) \\
\end{array}$ \\
\hline & Desorden Físico MB & $\begin{array}{l}-0.0372 \\
(0.1211) \\
\end{array}$ & - & - & $\begin{array}{l}- \\
-\end{array}$ & - \\
\hline & Comercio MB & $\begin{array}{r}0.56357^{* * *} \\
(0.0018)\end{array}$ & $\begin{array}{r}0.55629^{* * *} \\
(0.0014)\end{array}$ & $\begin{array}{r}0.54545^{* * *} \\
(0.0017)\end{array}$ & $\begin{array}{r}0.55103^{* * *} \\
(0.0015)\end{array}$ & $\begin{array}{r}0.52940^{* * *} \\
(0.0023)\end{array}$ \\
\hline & Educación MB & $\begin{array}{l}0.22036 \\
(0.1968) \\
\end{array}$ & $\begin{array}{r}0.19503 \\
(0.2395) \\
\end{array}$ & - & $\begin{array}{r}- \\
-\end{array}$ & - \\
\hline & Salud MB & $\begin{array}{r}0.1968 \\
(0.1413) \\
\end{array}$ & $\begin{array}{r}0.2395 \\
(0.1532) \\
\end{array}$ & - & - & - \\
\hline
\end{tabular}




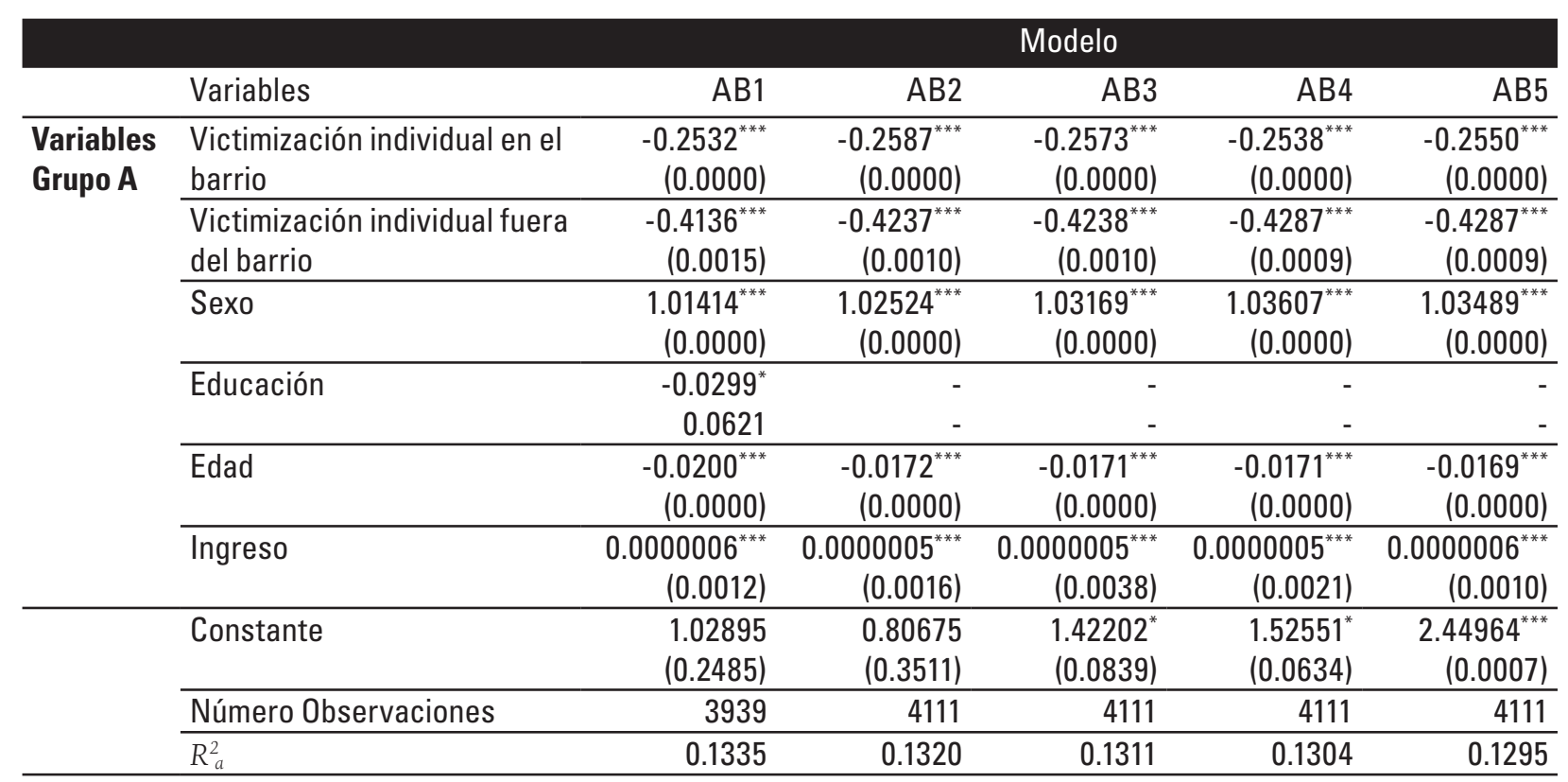

NOTA 1: Las variables terminadas en MB son promedios a nivel de microbarrio, las que no incluyen dicha clasificación son variables individuales de cada entrevistado.

NOTA 2: Los números entre paréntesis corresponden a los p-value de los coeficientes estimados en la regresión, los otros valores corresponden a los coeficientes de la regresión lineal, los cuales muestran en cuánto se ve afectada la variable dependiente (percepción de seguridad) ante variaciones de la variable explicativa. Los espacios en blanco corresponden a variables que no fueron incluidas en el modelo respectivo.

*** Estadísticamente significativo al 1\%

"Estadísticamente significativo al 5\%

"Estadísticamente significativo al 10\% 
Para las variables incluidas en el nivel comunitario, se observa que la antigüedad del barrio y la confianza promedio, junto con la interacción y la cohesión entre vecinos del barrio, afectan todas de manera positiva y significativa la percepción de seguridad, lo cual se mantiene ante cambios en la especificación del modelo (haciendo variar los regresores o variables explicativas).

Ahora bien, llama la atención que la variable disposición a actuar en comunidad, a pesar de que su nivel de significancia no es muy relevante (solo al 5\%), reporte un signo negativo, lo que implicaría que a mayor disposición a actuar en comunidad, las personas refieren menores niveles de seguridad. Por su parte, la variable control social informal, que es significativa (al 1\%), también reporta un signo negativo, del tal forma que las personas que refieren percibir mayores grados de control social informal en su barrio, se sienten menos seguras. Esta situación es contradictoria a lo propuesto por algunos estudios previos señalados en la revisión bibliográfica. Lo anterior puede deberse a problemas de simultaneidad o de causalidad inversa entre el control social informal y la percepción de seguridad, ya que no es claro que el control social informal determine unívocamente a la variable a explicar. Puede ser que, al mismo tiempo que esto ocurre, la percepción de seguridad de un individuo también afecte al control social informal,

ARTÍCULO: Determinantes individuales y del entorno residencial en la percepción de seguridad en barrios del Gran Santiago, Chile / Javier Núñez, Ximena Tocornal, Pablo Henríquez generándose así un problema de simultaneidad que, dada la naturaleza de los datos, esimposible de resolver.

La evaluación de programas de seguridad ciudadana a nivel municipal y la percepción de que los servicios básicos acudirán ante emergencias, poseen coeficientes positivos y significativos, lo cual indica que, contrario al control social informal, las redes de protección formales (como bomberos, emergencias, ambulancias, carabineros o seguridad ciudadana) sí están ayudando a hacer que las personas se sientan más seguras. Ninguna otra medición de presencia del Estado, como por ejemplo en salud o educación, resultan significativas, no obstante tienen los coeficientes esperados. Por su parte, la presencia de comercio de primera necesidad resulta significa en todos los casos, de tal modo que donde hay más almacenes, panaderías y otros puestos de este tipo, las personas se sienten más seguras.

Las señales de desorden físico en el barrio (captadas por un observador externo y entrenado) no resulta una medida que afecte significativamente la percepción de seguridad individual, al menos en presencia de las otras variables sociales, lo cual es consistente con lo encontrado por Ross y Jang, en tanto que las relaciones sociales permiten amortiguar (o en este caso, eliminar) el efecto que tiene el desorden físico sobre la percepción de seguridad. ${ }^{41}$

41 No obstante, el efecto encontrado por Ross y Jang, 2000, se da al consultar por percepciones de desorden físico y no por medidas objetivas como las utilizadas en este trabajo.

revista invi № 74 / Mayo 2012 / Volumen № 27: 87-120 113 
TABLA 4: EFECTOS DE VARIABLES PERSONALES SOCIODEMOGRÁFICAS (GRUPO A), DE RELACIONES SOCIALES Y TERRITORIALES (GRUPO B) Y DE DELITO, VIOLENCIA E INCIVILIDADES (GRUPO C) EN PERCEPCIÓN DE SEGURIDAD.

\begin{tabular}{|c|c|c|c|c|c|c|}
\hline \multicolumn{7}{|c|}{ Modelo } \\
\hline & Variables & $\mathrm{ABC1}$ & $\mathrm{ABC2}$ & $\mathrm{ABC} 3$ & $\mathrm{ABC} 4$ & $\mathrm{ABC5}$ \\
\hline \multirow{2}{*}{$\begin{array}{l}\text { Variables } \\
\text { Grupo C }\end{array}$} & Incivilidades MB & $\begin{array}{r}-0.0545^{* * *} \\
(0.0008)\end{array}$ & $\begin{array}{r}-0.0547^{* * *} \\
(0.0004)\end{array}$ & $\begin{array}{r}-0.0592^{* * *} \\
(0.0000)\end{array}$ & $\begin{array}{r}-0.0644^{* * *} \\
(0.0000)\end{array}$ & $\begin{array}{r}-0.0638^{* * *} \\
(0.0000)\end{array}$ \\
\hline & Victimización MB & $\begin{array}{l}3.1351^{* * *} \\
(0.0000)\end{array}$ & $\begin{array}{l}3.1316^{* * *} \\
(0.0000)\end{array}$ & $\begin{array}{c}3.1076^{* * *} \\
(0.0000)\end{array}$ & $\begin{array}{c}3.1302^{* * *} \\
(0.0000)\end{array}$ & $\begin{array}{c}2.9215^{* * *} \\
(0.0000)\end{array}$ \\
\hline \multirow{12}{*}{$\begin{array}{l}\text { Variables } \\
\text { Grupo B }\end{array}$} & Antigüedad MB & $\begin{array}{r}0.02701^{* * * *} \\
(0.0013)\end{array}$ & $\begin{array}{r}0.02917^{* * * *} \\
(0.0003)\end{array}$ & $\begin{array}{r}0.02950^{* * *} \\
(0.0002)\end{array}$ & $\begin{array}{r}0.03059^{* * *} \\
(0.0001)\end{array}$ & $\begin{array}{r}0.02925^{* * *} \\
(0.0002)\end{array}$ \\
\hline & Confianza MB & $\begin{array}{r}0.62218^{* * *} \\
(0.0000)\end{array}$ & $\begin{array}{r}0.52308^{* * * *} \\
(0.0000)\end{array}$ & $\begin{array}{r}0.52490^{* * *} \\
(0.0000)\end{array}$ & $\begin{array}{r}0.50247^{* * *} \\
(0.0000)\end{array}$ & $\begin{array}{r}0.49409^{* * *} \\
(0.0000)\end{array}$ \\
\hline & Cohesión MB & $\begin{array}{l}0.01866 \\
(0.7236) \\
\end{array}$ & $\begin{array}{r}0.0138 \\
(0.7864) \\
\end{array}$ & - & - & - \\
\hline & Disp. Actuar en Comunidad MB & $\begin{array}{r}-0.0479^{* *} \\
(0.0305)\end{array}$ & $\begin{array}{l}-0.0419^{*} \\
(0.0512)\end{array}$ & $\begin{array}{r}-0.0427^{* *} \\
(0.0456)\end{array}$ & $\begin{array}{r}-0.0114 \\
(0.5377)\end{array}$ & - \\
\hline & Interacción MB & $\begin{array}{r}0.06602^{* *} \\
(0.0187) \\
\end{array}$ & $\begin{array}{r}0.07857^{* * *} \\
(0.0037) \\
\end{array}$ & $\begin{array}{r}0.07758^{* * *} \\
(0.0032) \\
\end{array}$ & - & $\begin{array}{r}0.05144^{* *} \\
(0.0244) \\
\end{array}$ \\
\hline & Control Social Informal MB & $\begin{array}{r}-0.0705^{* * *} \\
(0.0000)\end{array}$ & $\begin{array}{r}-0.0661^{* * *} \\
(0.0000)\end{array}$ & $\begin{array}{r}-0.0692^{* * * *} \\
(0.0000)\end{array}$ & $\begin{array}{r}-0.0737^{* * *} \\
(0.0000)\end{array}$ & $\begin{array}{r}-0.0757^{* * *} \\
(0.0000)\end{array}$ \\
\hline & Seguridad Ciudadana MB & $\begin{array}{r}0.51987^{* * *} \\
(0.0000)\end{array}$ & $\begin{array}{r}0.57751^{* * *} \\
(0.0000)\end{array}$ & $\begin{array}{r}0.59414^{* * *} \\
(0.0000)\end{array}$ & $\begin{array}{r}0.58647^{* * *} \\
(0.0000)\end{array}$ & $\begin{array}{r}0.55800^{* * *} \\
(0.0000)\end{array}$ \\
\hline & Acuden MB & $\begin{array}{r}0.19960^{* * *} \\
(0.0000) \\
\end{array}$ & $\begin{array}{r}0.20401^{* * *} \\
(0.0000) \\
\end{array}$ & $\begin{array}{r}0.20005^{* * *} \\
(0.0000) \\
\end{array}$ & $\begin{array}{r}0.20182^{* * *} \\
(0.0000) \\
\end{array}$ & $\begin{array}{r}0.20446^{* * *} \\
(0.0000) \\
\end{array}$ \\
\hline & Desorden Físico MB & $\begin{array}{r}-0.0310 \\
(0.1959)\end{array}$ & $\begin{array}{c}-0.0293 \\
(0.2079) \\
\end{array}$ & - & - & - \\
\hline & Comercio MB & $\begin{array}{r}0.46234^{*} \\
(0.0102)\end{array}$ & $\begin{array}{r}0.46960^{* * *} \\
(0.0069)\end{array}$ & $\begin{array}{r}0.45062^{* * *} \\
(0.0092)\end{array}$ & $\begin{array}{r}0.46806^{* * *} \\
(0.0068)\end{array}$ & $\begin{array}{r}0.45965^{* * *} \\
(0.0079)\end{array}$ \\
\hline & Presencia Estado Educación MB & $\begin{array}{r}0.1580 \\
(0.3580) \\
\end{array}$ & $\begin{array}{r}- \\
-\end{array}$ & - & - & - \\
\hline & Presencia Estado Salud MB & $\begin{array}{r}0.1908 \\
(0.2005)\end{array}$ & - & $\begin{array}{l}- \\
-\end{array}$ & - & $\begin{array}{l}- \\
-\end{array}$ \\
\hline
\end{tabular}




\begin{tabular}{|c|c|c|c|c|c|c|}
\hline \multicolumn{7}{|c|}{ Modelo } \\
\hline & Variables & $\mathrm{ABC1}$ & $\mathrm{ABC} 2$ & ABC3 & $\mathrm{ABC} 4$ & $\mathrm{ABC5}$ \\
\hline \multirow{10}{*}{$\begin{array}{l}\text { Variables } \\
\text { Grupo A }\end{array}$} & Victimización individual en el barrio & $\begin{array}{r}-0.2193^{* * *} \\
(0.0000)\end{array}$ & $\begin{array}{r}-0.2231^{* * *} \\
(0.0000)\end{array}$ & $\begin{array}{r}-0.2236^{* * *} \\
(0.0000)\end{array}$ & $\begin{array}{r}-0.2247^{* * *} \\
0.0000\end{array}$ & $\begin{array}{r}-0.2221^{* * *} \\
0.0000\end{array}$ \\
\hline & $\begin{array}{l}\text { Victimización individual fuera del } \\
\text { barrio }\end{array}$ & $\begin{array}{r}-0.3687^{* * *} \\
(0.0045)\end{array}$ & $\begin{array}{r}-0.3786^{* * *} \\
(0.0031)\end{array}$ & $\begin{array}{r}-0.3770^{* * *} \\
(0.0033)\end{array}$ & $\begin{array}{r}-0.3833^{* * *} \\
(0.0028)\end{array}$ & $\begin{array}{r}-0.3819^{* * *} \\
(0.0029)\end{array}$ \\
\hline & Sexo & $\begin{array}{r}1.00149^{* * *} \\
(0.0000)\end{array}$ & $\begin{array}{r}1.01540^{* * *} \\
(0.0000)\end{array}$ & $\begin{array}{r}1.01470^{* * *} \\
(0.0000)\end{array}$ & $\begin{array}{r}1.01053^{* * *} \\
(0.0000)\end{array}$ & $\begin{array}{r}1.01810^{* * *} \\
(0.0000)\end{array}$ \\
\hline & Fducrción & $-0.0326^{* *}$ & - & - & - & - \\
\hline & Euucacion & $(0.0422)$ & - & - & - & - \\
\hline & Edad & $\begin{array}{r}-0.0208^{* * *} \\
(0.0000) \\
\end{array}$ & $\begin{array}{r}-0.0177^{* * *} \\
(0.0000) \\
\end{array}$ & $\begin{array}{r}-0.0177^{* * *} \\
(0.0000) \\
\end{array}$ & $\begin{array}{r}-0.0177^{* * *} \\
(0.0000) \\
\end{array}$ & $\begin{array}{r}-0.0178^{* * *} \\
(0.0000) \\
\end{array}$ \\
\hline & Ingreso & $\begin{array}{r}0.0000004^{* *} \\
(0.0285)\end{array}$ & $\begin{array}{r}0.0000003^{*} \\
(0.0933)\end{array}$ & $\begin{array}{r}0.0000003^{*} \\
(0.0784)\end{array}$ & $\begin{array}{r}0.0000002 \\
(0.1214)\end{array}$ & $\begin{array}{r}0.0000003^{*} \\
(0.0611)\end{array}$ \\
\hline & Constante & $\begin{array}{r}5.30770^{* * *} \\
(0.0000)\end{array}$ & $\begin{array}{r}5.66095^{* * *} \\
(0.0000)\end{array}$ & $\begin{array}{r}5.92591^{* * *} \\
(0.0000)\end{array}$ & $\begin{array}{r}7.14495^{* * *} \\
(0.0000)\end{array}$ & $\begin{array}{r}6.10173^{* * *} \\
(0.0000)\end{array}$ \\
\hline & Número Observaciones & 3939 & 4111 & 4111 & 4111 & 4111 \\
\hline & $R_{a}^{2}$ & 0.1417 & 0.1398 & 0.1399 & 0.1383 & 0.1393 \\
\hline
\end{tabular}

NOTA 1: Las variables terminadas en MB son promedios a nivel de microbarrio, las que no incluyen dicha clasificación son variables individuales de cada jefe de hogar.

NOTA 2: Los números entre paréntesis corresponden a los p-value de los coeficientes estimados en la regresión, los otros valores corresponden a los coeficientes de la regresión lineal, los cuales muestran en cuánto se ve afectada la variable dependiente (percepción de seguridad) ante variaciones de la variable explicativa. Los espacios en blanco corresponden a variables que no fueron incluidas en el modelo respectivo.

*** Estadísticamente significativo al 1\%

"Estadísticamente significativo al 5\%

"Estadísticamente significativo al 10\% 


\section{MODELO ABC: REGRESIONES CON TODAS LAS VARIABLES}

Como se comentó en la sección anterior, el modelo general incluye todos los grupos de variables analizados, para ver cómo varían los resultados con respecto a la situación en que solo están algunos de ellos. Esto permite detectar el posible doble efecto que tienen las variables de relaciones sociales, tanto de manera directa, como indirecta, a través de la generación de incivilidades y violencia. La tabla 4 muestra las estimaciones por Mco del modelo general.

Es posible observar que el haber sido víctima de delito dentro o fuera del barrio en los últimos 12 meses mantiene el signo, magnitud y significancia encontrado en las estimaciones previas. Esto permite afirmar que la victimización afecta negativamente la percepción de seguridad dentro del barrio, sin importar dónde se ha sido víctima de un delito. Ello podría implicar que para las personas victimizadas en otros lugares de la ciudad, la inseguridad no distingue límites territoriales entre el barrio y los otros territorios urbanos donde se ha sido victimizado, atribuyéndole eventualmente mayor peligrosidad al entorno residencial cotidiano del que posiblemente éste amerita. El temor al delito parecería ser un fenómeno que no se puede circunscribir a un determinado territorio en función de experiencias previas de victimización delictual.

116 revista invi № 74 / Mayo 2012 / Volumen № 27: 87-120
Asimismo, la tasa de victimización del barrio continúa siendo significativa al 1\% en todos los casos y afectando negativamente la percepción de seguridad. Esto muestra la relevancia de la victimización vicaria, es decir, que no es necesario haber sido víctima de un delito para estar inseguro dentro del barrio, sino que bastaría que los vecinos hayan sido victimizados, para disminuir la percepción de seguridad. Lo anterior es concordante con los estudios previos señalados y también se relaciona con el nivel de interacción entre vecinos ya que a mayor interacción entre vecinos, sería esperable una mayor difusión de los eventos delictuales ocurridos en el barrio y posibles reacciones defensivas o preactivas de los vecinos para lidiar con las amenazas delictuales. De hecho, las medidas de interacción entre vecinos en el modelo general resultan significativas en un $1 \%$ o en $5 \%$ dependiendo de las variables incluidas.

Las incivilidades se mantienen significativas al 1\% afectando negativamente la percepción de seguridad. No obstante, su coeficiente pierde la mitad de su magnitud con respecto al modelo AC, lo que podría estar confirmando el efecto que tienen sobre las incivilidades las variables sociales. En efecto, las variables sociales continúan siendo significativas y conservan la fuerza de sus coeficientes, especialmente, antigüedad, confianza y control social informal. Sin embargo, la cohesión pierde su significancia al introducirse en el modelo 
las variables de percepción de incivilidades y tasa de victimización del barrio y, en menor grado, algo similar ocurre con la disposición a actuar en comunidad, que es significativa solo al $5 \%$ o al $10 \%$ dependiendo de los casos.

La variable de antigüedad del barrio, se mantiene significativa al $1 \%$ y afecta positivamente la seguridad, mostrando el rol que tiene el tiempo en la generación de seguridad. Tanto la confianza en los vecinos como la interacción vecinal, disminuyen de manera significativa la inseguridad, lo cual muestra el importante rol que posee el confiar entre vecinos e interactuar con ellos, no obstante, la cohesión pierde significancia. En otras palabras, deja de ser relevante que los vecinos consideren que hay homogeneidad y unión en el territorio para que se genere mayor seguridad en presencia de variables directamente relacionadas con delitos y percepción de violencia e incivilidades.

El control social informal mantiene su significancia al 1\%, mientras la disposición a actuar en comunidad solo a un 5\% o 10\%. Lo llamativo es que ambas variables permanecen con signos inversos a los esperados, de tal forma que a más control social informal y mayor disposición a actuar en comunidad, las personas se sentirían más inseguras. Más allá de los problemas metodológicos para poder desentrañar esta situación, lo interesante es que desafía los entendimientos esperados. Es posible que los mayores niveles de control social informal en un barrio se deban precisamente a situaciones amenazantes ante las cuales los vecinos deben reaccionar y ello pasa por adquirir socialmente un cierto nivel de conciencia de los problemas comunes que podría estar haciendo disminuir la percepción de seguridad.

Con respecto a la percepción de que los servicios básicos acudan en una emergencia, así como también la evaluación de los programas de seguridad municipales, se aprecia que continúan siendo variables significativas. Esto pone en relieve que, al menos del lado de la percepción de seguridad ciudadana, se justificaría la existencia de estos programas. Las variables de presencia del Estado y de desorden físico, continúan sin ser significativas, pero de signo esperado.

La presencia de comercio básico en el barrio también continúa teniendo un efecto positivo sobre la percepción de seguridad, debido posiblemente a que genera relaciones sociales e interacciones cotidianas. El comercio puede ser visto como un lugar donde los vecinos se conocen y se encuentran. A la vez, puede haber un efecto de "votación con los pies", vale decir, es probable que los comerciantes elijan los lugares más seguros para instalarse, por lo cual correlacionaría la percepción de seguridad de los vecinos, con la existencia de este tipo de comercio. 
La variable género sigue indicando el menor reporte de seguridad entregado por las mujeres, asimismo podemos ver que a mayor edad disminuye la seguridad. El nivel de ingreso es no relevante estadísticamente, al igual que los años de educación, situación que se repitió en todas las estimaciones realizadas, manteniéndose casi constantes las magnitudes y significancias de los coeficientes.

\section{Conclusiones}

Este trabajo desarrolla una metodología empírica multinivel basada en regresiones lineales multivariadas para estudiar el rol que juegan tanto las características individuales como las características del entorno residencial de los individuos sobre las percepciones de (in)seguridad de las personas. El estudio encuentra evidencia del rol que juegan diversas características individuales sobre la percepción de inseguridad, en particular género, edad y experiencias recientes de victimización, de manera coherente con la evidencia previa. Adicionalmente, se encuentra evidencia de diversas variables asociadas a los atributos de la interacción social en el barrio que inciden en la percepción de inseguridad. En particular, se encontró que las medidas de confianza, cohesión e interacción entre vecinos junto a la antigüedad de los barrios están generalmente asociadas a menores niveles de percepción de inseguridad. También se proporciona evidencia de que los niveles promedio de victimización y percepción de violencia e incivilidades en los barrios se asocian a mayores niveles de temor de sus habitantes (separadamente del efecto de las experiencias individuales recientes de victimización). Estos resultados son sugerentes de que la calidad de la interacción social que impera entre los vecinos es un factor de primera importancia en la configuración de las percepciones de temor e inseguridad de la población.

Por otra parte, la presencia de programas de seguridad ciudadana a nivel municipal así como la percepción de los vecinos de que a su barrio acudirían vehículos y personal de primeros auxilios y de servicios básicos tienen un efecto positivo sobre la seguridad de las personas en sus entornos residenciales. Estos resultados sugieren que este tipo de políticas e instituciones son importantes y justificables no sólo por sus efectos sobre los niveles efectivos de criminalidad y victimización, sino que también en los niveles percibidos de temor e inseguridad que experimenta la población.

La existencia de desorden físico en el barrio, aunque posee en general el signo esperado de asociarse a mayores niveles percibidos de inseguridad, no posee un efecto significativo una vez que otras variables son incorporadas. Y también llama la atención que las medidas de disposición a actuar en comunidad y de control social informal poseen a menudo efectos inversos a los esperados. Una explicación es la posible existencia de endogeneidad 
o de causalidad inversa entre estas variables y los niveles de temor. Es posible, por ejemplo, que mayores niveles de inseguridad motiven a la población a actuar en comunidad y a establecer mecanismos de control social informal para hacer frente a la violencia y la victimización en los barrios, hipótesis que amerita mayor investigación a futuro.

Por último cabe señalar que este estudio se basa en una base de datos de corte transversal, de manera tal que los resultados obtenidos deben ser tomados con cautela. En este contexto, es necesario avanzar en la dirección de generar estudios y evidencia a partir de datos de panel o longitudinales de entornos residenciales que permitan reexaminar los efectos estudiados en esta investigación. Esto permitirá obtener evidencia más abundante y robusta sobre los determinantes de la percepción de inseguridad y las intervenciones y políticas públicas que pueden diseñarse e implementarse en esa materia.

\section{Bibliografía}

BRUNO, F. J. Diccionario de términos psicológicos fundamentales. 1997. Barcelona. Paidós Studio.

CRAWFORD, Adam. Questioning Appeals to Community within Crime Prevention and Control European Journal on Criminal Policy and Research. 7(4): 509-530, 1999, DOI: 10.1023/A:1008793906097.

ARTíCULO: Determinantes individuales y del entorno residencial en la percepción de seguridad en barrios del Gran Santiago, Chile / Javier Núñez, Ximena Tocornal, Pablo Henríquez
BRUNTON-SMITH, lan and STURGIS, Patrick. Do Neighborhoods Generate Fear Of Crime? An Empirical Test Using the British Crime Survey. Criminology. 49 (2): 331-369, 2011. ISSN 0011-1384.

DAMMERT, Lucía. Perspectivas y dilemas de la seguridad ciudadana en América Latina. Quito, Ecuador, FLACSO-Ecuador. 2007. 334 p. ISBN 9789978-67145-0.

DAMMERT, Lucía. ¿Ciudad sin Ciudadanos? Fragmentación, Segregación y Temor en Santiago. Eure, 30 (091): 87-96, 2004. ISSN 0250-7161.

DAMMERT, Lucía; LUNECKE, Alejandra. Victimización y temor en Chile: Revisión teórico-empírica en Doce Comunas del País. Serie de Estudios CESC, Instituto de Asuntos Públicos, Universidad de Chile. 2002. 59 p. ISBN 956-19-0391-1

DAMMERT, Lucía; MALONE, Mary. Does It Take a Village? Policing Strategies and Fear of Crime in Latin America. Latin American Politics and Society 48(4): 27-51, 2006 ISSN: 1548-2456.

HUMMELSHEIM, Dina; HIRTENLEHNER, Helmut; JACKSON, Jonathan and OBERWITTLER, Dietrich. Social Insecurities and Fear of Crime: A Cross-National Study on the Impact of Welfare State Policies on Crime-related Anxieties. European Sociological Review. 27 (3): 327-345, 2010.

PAIN, Rachel. Place, social relations and the fear of crime: a review. Progress in Human Geography 24(3): 365-387, 2000.

revista invi $\mathrm{N}^{0}$ 74 / Mayo 2012 / Volumen № 27: 87-120 119 
RAUDENBUSH, Stephen, SAMPSON Robert. Econometrics: Toward a science of assessing ecological settings, with application to the systematic social observation of neighborhoods. Sociological Methodology. 29(1): 1-41, 1999.

ROCCATO, Michele; RUSSO Silvia and VIENO, Alessio. Perceived community disorder moderates the relation between victimization and fear of crime. Journal of Community Psychology. 39 (7): 884888, 2011. ISSN 0090-4392.

ROSS, Catherine; JANG Sung Joon. Neighborhood Disorder,Fear, andMistrust:TheBufferingRoleofSocial Ties with Neighbors. American Journal of Community Psychology 28(4): 401-20, 2000. ISSN 0091-0562.

RUIZ PÉREZ, José Ignacio. Eficacia colectiva, cultura ciudadana y victimización: un análisis exploratorio sobre sus relaciones con diversas medidas del miedo al crimen. Acta Colombiana de Psicología, 13 (1): 103-114, 2010.

SAMPSON, Robert; MCADAM, Doug; MACINDOE, Heather; WEFFER-ELIZOND0, Simón. Civil society reconsidered: The durable nature and community structure of collective civic action. American Journal of Sociology. 111(3): 673-714, 2005.
SAMPSON Robert; RAUDENBUSH, Stephen. Systematic social observation of public spaces: A new look at disorder in urban neighborhoods. American Journal of Sociology. 105(3): 603-651, 1999.

SAMPSON Robert; RAUDENBUSH, Stephen; EARLS, Felton. Neighborhoods and violent crime: A multilevel study of collective efficacy. Science. 277(5328): 918-924, August 1997.

VILALTA, Carlos. Fear of crime in gated communities and apartment buildings: a comparison of housing types and a test of theories. Journal of Housing and the Built Environment. 26(2): 107-121, 2011.

VOZMEDIANO, Laura; SAN JUAN, César y VERGARA, Ana Isabel. Problemas de medición del miedo al delito: Algunas respuestas teóricas y técnicas. (En línea). Revista Electrónica de Ciencia Penal y Criminología. (10): 07:1-07:1.7. 2008 [Fecha de consulta: 7 noviembre 2011]. Disponible en: http:// criminet.ugr.es/recpc/10/recpc10-07.pdf. ISSN 1695-0194.

WILSON, James; KELLING, George L. Broken Windows: The Police and Neighborhood Safety. Atlantic Monthly. 1982. 